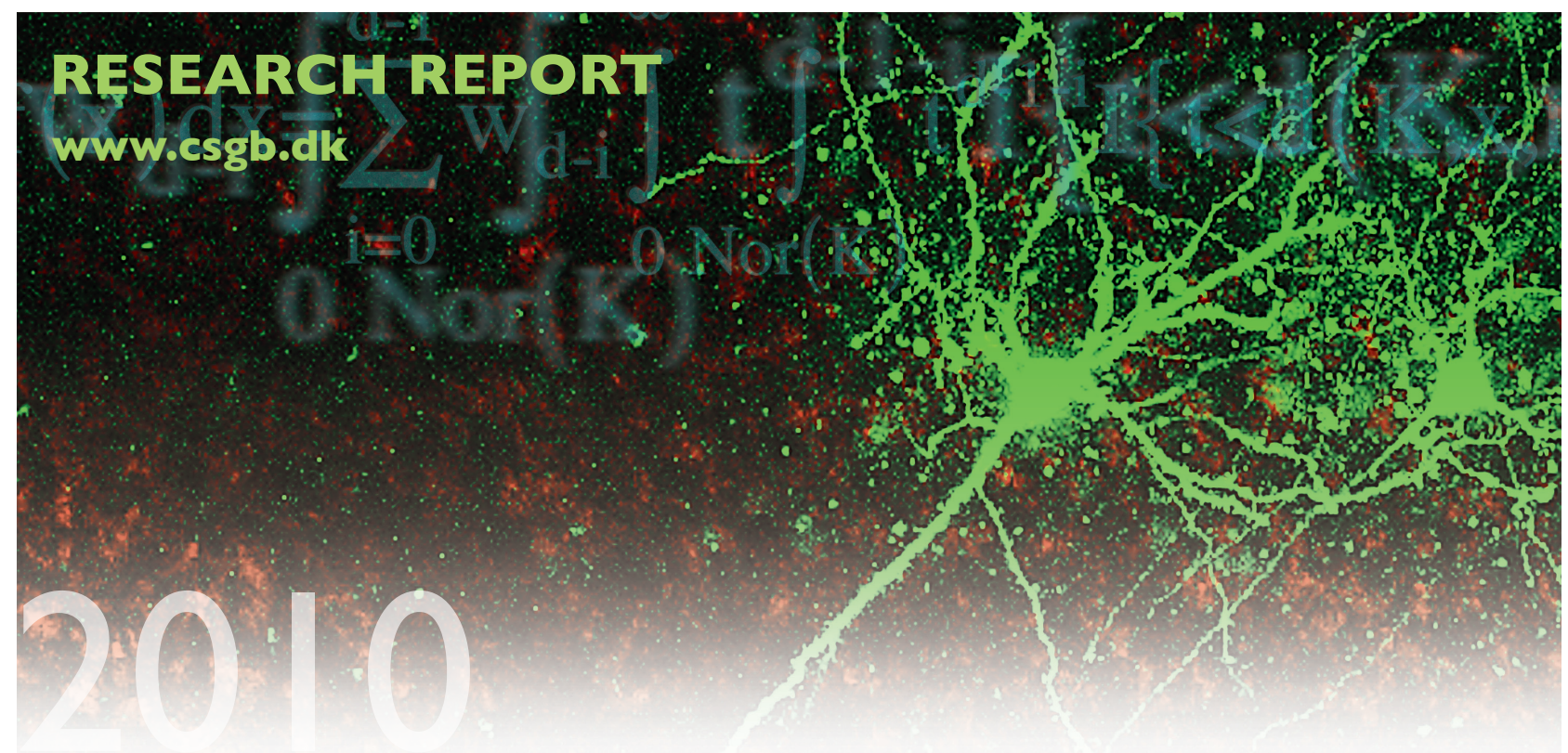

Jürgen Kampf

The parallel volume at large distance

No. 01 , May 2010 


\title{
The parallel volume at large distances
}

\author{
Jürgen Kampf, \\ Department of Mathematical Sciences, Aarhus University
}

\begin{abstract}
In this paper we examine the asymptotic behavior of the parallel volume of planar non-convex bodies as the distance tends to infinity. We show that the difference between the parallel volume of the convex hull of a body and the parallel volume of the body itself tends to 0 . This yields a new proof for the fact that a planar body can only have polynomial parallel volume, if it is convex. Extensions to Minkowski spaces and random sets are also discussed.
\end{abstract}

\section{Introduction}

The parallel body of a body $K$ at distance $r \geq 0$ is the set of all points having at most distance $r$ from $K$. Its volume is called the parallel volume of $K$ at distance $r$. The parallel volume is a functional which plays an important role in convex geometry and has many applications, e.g. in stochastic geometry, statistics, discrete geometry and geometric functional analysis. While in many applications the parallel volume of arbitrary bodies is interesting, it has been explored mainly in the special case of convex bodies.

We put

$$
\begin{aligned}
K+L & :=\{x+y \mid x \in K, y \in L\}, & & K, L \in \mathcal{C}, \\
r K & :=\{r x \mid x \in K\}, & & K \in \mathcal{C}, r \in \mathbb{R}_{0}^{+},
\end{aligned}
$$

where $\mathcal{C}$ denotes the set of all bodies in $\mathbb{R}^{d}$, i.e. of all non-empty compact sets, and $\mathbb{R}_{0}^{+}$denotes the set of non-negative real numbers. Now it is easy to see that the parallel body of a body $K$ at distance $r \geq 0$ is $K+r B^{d}$, where $B^{d}$ denotes the $d$ dimensional closed unit ball. By $V_{d}$ we denote the $d$-dimensional Lebesgue-measure and by conv $K$ we denote the convex hull of a body $K$.

Steiner [10] was probably the first to consider the parallel volume of a given body as a functional of the distance, when he showed in 1840 that the parallel volume of certain convex bodies is a polynomial. Meanwhile it is known that this is true for all convex bodies. In 1935 Lusternik [7] proved the Brunn-Minkowski-inequality

$$
V_{d}(K+r B) \geq\left(\sqrt[d]{V_{d}(K)}+r \sqrt[d]{V_{d}(B)}\right)^{d}, \quad r \geq 0,
$$

for arbitrary bodies $K, B \subseteq \mathbb{R}^{d}$. In the 1950s Kneser [6] and Sz.-Nagy [11] obtained further inequalities, which relate the parallel volume a fixed convex body has at various distances to each other. 
In this paper we want to examine the asymptotic behavior of the parallel volume as the distance tends to infinity. Our main theorem is

Theorem 1.1. Let $K \subseteq \mathbb{R}^{2}$ be a body. Then

$$
\lim _{r \rightarrow \infty} V_{2}\left((\operatorname{conv} K)+r B^{2}\right)-V_{2}\left(K+r B^{2}\right)=0 .
$$

This leads to a new proof of the following theorem by Heveling, Hug and Last [2]:

Theorem 1.2. Let $K \subseteq \mathbb{R}^{2}$ be a body, for which

$$
\mathbb{R}_{0}^{+} \rightarrow \mathbb{R}_{0}^{+}, r \mapsto V_{2}\left(K+r B^{2}\right)
$$

is a polynomial. Then $K$ is convex.

Hug, Last and Weil [3] extended this result to random bodies and more general 2 -dimensional normed spaces. They also gave an interpretation for bodies $K$ having more than 2 dimensions.

This paper is organized as follows:

In Section 2 we collect some results from convex and Minkowski geometry we need in the later sections.

In Section 3 of this paper we state and prove a more general version of Theorem 1.1. We will show that, when one replaces $B^{2}$ by another convex body $B \subseteq \mathbb{R}^{2}$, the theorem remains true, iff $B$ is smooth (in the sense we define it in section 3 ). We will also prove this statement for random bodies and give an interpretation for bodies $K$ having more than 2 dimensions.

In section 4 we give a new proof for Theorem 1.2 under generalisations quite similar to those of [3].

In section 5 we show that if a union of randomly many random bodies fulfilling certain regularity assumptions has polynomial expected parallel volume, then this union consists of only one body a.s.

In section 6 we will give an outlook on work in progress about the speed of convergence in (1).

\section{Geometric tools}

In this section we will collect results from convex and Minkowski geometry that will be used in sections 3 and 4 .

For $K \in \mathcal{C}, B \in \mathcal{K}$ and $r \geq 0$ we can write the expression conv $K+r B$ without brackets, since as a special case of [8, Theorem 1.1.2] we have

$$
\operatorname{conv}(K+r B)=(\operatorname{conv} K)+r B .
$$

It is well-known (see e.g. [8, section 5.1]) that for convex bodies $K, B \subseteq \mathbb{R}^{d}$ there are numbers $V(K[d-j], B[j]), j=0, \ldots, d$, called mixed volumes, such that

$$
V_{d}(K+r B)=\sum_{j=0}^{d}\left(\begin{array}{l}
d \\
j
\end{array}\right) r^{j} V(K[d-j], B[j]), \quad r \geq 0 .
$$


In this paper we consider $\mathcal{K}$ always with the Fell-Matheron- $\sigma$-algebra, which is known to be the Borel- $\sigma$-algebra of the Hausdorff-metric. For a introduction, see e.g. [9, chapter 12].

Let $\mathcal{K}^{2 \|}$ denote the set of all pairs $(K, B)$ of two 2-dimensional convex bodies in $\mathbb{R}^{n}$ that have parallel affine hulls. We will show the measurability of $\mathcal{K}^{2 \|}$ in Lemma A.4.

Lemma 2.1. For $(K, B) \in \mathcal{K}^{2 \|}$ and $r \geq 0$ the body $K+r B$ is 2-dimensional. There is a measurable map $V: \mathcal{K}^{2 \|} \rightarrow \mathbb{R}$ such that

$$
V_{2}(K+r B)=V_{2}(K)+2 r V(K, B)+r^{2} V_{2}(B)
$$

for all $r \geq 0$ and $(K, B) \in \mathcal{K}^{2 \|}$.

Proof. In the special case $n=2$ this statement is well known (see e.g. [8, Theorem 5.1.6]). If $n>2$ and $(K, B) \in \mathcal{K}^{2 \|}$, choose an isometry $\alpha: \hat{K} \rightarrow \mathbb{R}^{2}$ and a translation $\beta: \hat{B} \rightarrow \hat{K}$, where $\hat{K}$ and $\hat{B}$ are the affine hulls of $K$ and $B$. Set $V(K, B):=V(\alpha(K), \alpha \circ \beta(B))$. By the translation invariance and the joint rotation invariance of the mixed volume, this extension is well-defined. By the isometry invariance of the Lebesgue measure (2) remains true.

In order to see that $V$ is measurable, we prove that it is continuous. So let $\left(\left(K_{k}, B_{k}\right)\right)_{k \in \mathbb{N}^{+}}$be a sequence in $\mathcal{K}^{2 \|}$ converging to $(K, B) \in \mathcal{K}^{2 \|}$. Now it is possible to choose the isometries $\alpha_{k}: \hat{K}_{k} \rightarrow \mathbb{R}^{2}, \beta_{k}: \hat{B}_{k} \rightarrow \hat{K}_{k}, \alpha: \hat{K} \rightarrow \mathbb{R}^{2}$ and $\beta: \hat{B} \rightarrow \hat{K}$ in such a way that $\lim _{k \rightarrow \infty} \alpha_{k}\left(x_{k}\right)=\alpha(x)$, iff $\lim _{k \rightarrow \infty} x_{k}=x$, and $\lim _{k \rightarrow \infty} \alpha_{k}\left(\beta_{k}\left(y_{k}\right)\right)=$ $\alpha\left(\beta\left(\lim _{k \rightarrow \infty} y_{k}\right)\right)$, iff $\lim _{k \rightarrow \infty} y_{k}=y$, for all sequences $\left(x_{k}\right)_{k \in \mathbb{N}^{+}}$and $\left(y_{k}\right)_{k \in \mathbb{N}^{+}}$with $x_{k} \in \hat{K}_{k}$ and $y_{k} \in B_{k}$ for all $k \in \mathbb{N}^{+}$and points $x \in \hat{K}$ and $y \in \hat{B}$. Now it is easy to see that $\lim _{k \rightarrow \infty} \alpha_{k}\left(K_{k}\right)=\alpha(K)$ and $\lim _{k \rightarrow \infty} \alpha_{k}\left(\beta_{k}\left(B_{k}\right)\right)=\alpha(\beta(B))$. Since in the special case $n=2$ the functional $V$ is continuous by [8, Theorem 5.1.6], we have shown the continuity of $V$ (for arbitrary $n \geq 2$ ).

We denote the interior of a subset $A \subseteq \mathbb{R}^{d}$ by $\operatorname{int} A$ and its boundary by bd $A$.

In the rest of this section we let $B \subseteq \mathbb{R}^{d}$ be a convex body with $0 \in \operatorname{int} B$, called the gauge body. For a closed set $A \subseteq \mathbb{R}^{d}$ and $x \in \mathbb{R}^{d}$ we define the $B$-distance from $x$ to $A$ to be

$$
d_{B}(A, x):=\min \{t \geq 0 \mid x \in A+t B\} .
$$

For $x, y \in \mathbb{R}^{d}$ we put

$$
d_{B}(y, x):=d_{B}(\{y\}, x)
$$

Then it is easy to see that

$$
\begin{array}{ll}
d_{B}(x, y)=0 \Longleftrightarrow x=y, & x, y \in \mathbb{R}^{d}, \\
d_{B}(x+\lambda u, x+\lambda v)=\lambda d_{B}(u, v), & x, u, v \in \mathbb{R}^{d}, \lambda \in \mathbb{R}_{0}^{+}, \\
d_{B}(x, y)+d_{B}(y, z) \geq d_{B}(x, z), & x, y, z \in \mathbb{R}^{d},
\end{array}
$$

and that $d_{B}: \mathbb{R}^{d} \times \mathbb{R}^{d} \rightarrow \mathbb{R}_{0}^{+}$is continuous.

For a closed set $A \subseteq \mathbb{R}^{d}$ and $x \in \mathbb{R}^{d}$ we put

$$
\Pi_{B}(A, x):=\left\{y \in A \mid d_{B}(y, x)=d_{B}(A, x)\right\} .
$$


Lemma 2.2. Let $K \subseteq \mathbb{R}^{d}$ be a convex body and $x \in \mathbb{R}^{d} \backslash K$. Then a point $y \in K$ lies in $\Pi_{B}(K, x)$, iff there is a (Euclidean) exterior unit normal vector $u \in \mathbb{R}^{d}$ of $K$ in $y$, a point $q \in B$ and a number $s \in \mathbb{R}_{0}^{+}$such that $u$ is (Euclidean) exterior unit normal vector of $B$ in $q$ and $x=y+s q$. In this case we have $s=d_{B}(y, x)$.

Proof. Assume $\in \Pi_{B}(K, x)$. Put $r:=d_{B}(K, x)$. By the cancelation law for Minkowski sums (see [8, p. 46]) one can show $x \in \operatorname{bd}(K+r B)$. Choose an (Euclidean) exterior unit normal vector $u \in \mathbb{R}^{d}$ of $K+r B$ in $x$ and a point $q \in B$ with $x=y+r q$. Then for arbitrary $p \in B$ one has $y+r p \in K+r B$ and hence $\langle y+r p, u\rangle \leq\langle x, u\rangle$, which implies $\langle p, u\rangle \leq\langle q, u\rangle$. So $u$ is an exterior unit normal vector of $B$ in $q$ and in the same way one can show that $u$ is an exterior unit normal vector of $K$ in $y$.

Now assume that $u, q$ and $s$ as described in the lemma exist. Considering scalar products with $u$ one obtains $d_{B}(K, x) \geq s$. Since $d_{B}(y, x) \leq s$ we have $y \in \Pi_{B}(K, x)$.

Lemma 2.3. Let $K \subseteq \mathbb{R}^{d}$ be a convex body and $x \in \mathbb{R}^{d}$. Then $\Pi_{B}(K, x)$ is convex.

Proof. Put $r:=d_{B}(K, x)$. Let $y, y^{\prime} \in \Pi_{B}(K, x)$ and $\lambda \in[0,1]$. Then there are $q, q^{\prime} \in B$ such that $x=y+r q=y^{\prime}+r q^{\prime}$. Now we have $\lambda y+(1-\lambda) y^{\prime} \in K$, $\lambda q+(1-\lambda) q^{\prime} \in B$ and

$$
\lambda y+(1-\lambda) y^{\prime}+r \cdot\left(\lambda q+(1-\lambda) q^{\prime}\right)=\lambda(y+r q)+(1-\lambda)\left(y^{\prime}+r q^{\prime}\right)=x .
$$

Hence $\lambda y+(1-\lambda) y^{\prime} \in \Pi_{B}(K, x)$.

\section{The convergence result}

In this section we prove the main theorem of the present paper.

A convex body $B$ is called smooth, if for each point $y \in \mathrm{bd} K$ there is a unique exterior unit normal vector. By [8, p. 104] a convex body is smooth, iff its boundary is a $C^{1}$-manifold.

We call a 2-dimensional convex body which is smooth if considered as a subset of its affine hull a disc body. We denote the affine hull of a body $B$ by $\hat{B}$ and call

$$
B^{\perp}:=\left\{v \in \mathbb{R}^{n} \mid\langle v, y-x\rangle=0 \text { for all } x, y \in B\right\}
$$

the affine-orthogonal compliment of $B$. For two bodies $K, B \subseteq \mathbb{R}^{n}$ we let

$$
K_{B}:=\bigcup_{x \in B^{\perp}}(\operatorname{conv} K \cap(x+\hat{B}))
$$

denote the $B$-convexification of $K$. We denote by diam $A$ the diameter of a bounded subset $A \subseteq \mathbb{R}^{d}$ and by $\rho_{B}$ the biggest radius of a ball lying in a convex body $B \subseteq \mathbb{R}^{d}$ (a compactness argument ensures that there is a biggest one).

A random closed set is a measurable function taking values in the set of all closed subset of $\mathbb{R}^{d}$ equipped with the Fell-Matheron- $\sigma$-algebra (for details see e.g. [9, section 2.1 and 12.2]). A random body, convex body resp. disc body is a random closed set that a.s. takes values in the set of all bodies, convex bodies resp. disc bodies. 
By [9, Lemma 1.2.1 and Theorem 2.4.2] the sets $\mathcal{C}$ and $\mathcal{K}$ are measurable. In $[9$, section 12.3] it is shown that the functions $\mathcal{C} \times \mathcal{C} \rightarrow \mathcal{C},(K, L) \mapsto K+L$ and $\mathbb{R}_{0}^{+} \times \mathcal{C} \rightarrow \mathcal{C},(r, K) \mapsto r K$ and $V_{d}: \mathcal{C} \rightarrow \mathbb{R}_{0}^{+}$are measurable. The functions diam $: \mathcal{C} \rightarrow \mathbb{R}_{0}^{+}$and $\mathcal{K} \rightarrow \mathbb{R}_{0}^{+}, B \rightarrow \rho_{B}$ are Lipschitz-continuous w.r.t. the Hausdorff metric and hence measurable. The measurability of the sets $\mathcal{K}_{\text {Disc }}$ of all disc bodies and of $\mathcal{C} \times \mathcal{C} \rightarrow \mathcal{C},(K, B) \rightarrow K_{B}$ will be proven in the appendix.

Theorem 3.1. Let $X \subseteq \mathbb{R}^{n}$ be a random body and $Y \subseteq \mathbb{R}^{n}$ a random disc body such that $\mathbb{E} V_{n}\left(X_{Y}+Y\right)<\infty$ and

$$
\mathbb{E}\left[\left(V_{n}\left(X_{Y}+Y\right)-V_{n}\left(X_{Y}\right)\right) \cdot \operatorname{diam} X / \rho_{Y}\right]<\infty .
$$

Then

$$
\mathbb{E} V_{n}\left(X_{Y}+r Y\right)-\mathbb{E} V_{n}(X+r Y) \stackrel{r \rightarrow \infty}{\longrightarrow} 0 .
$$

Observe that $V_{n}\left(X_{Y}+r Y\right)$ in Theorem 3.1 is a.s. a polynomial in $r$ with nonnegative, measurable coefficents by 2.1. Hence the assumption $\mathbb{E} V_{n}\left(X_{Y}+Y\right)<\infty$ implies that $\mathbb{E} V_{n}\left(X_{Y}+r Y\right)$ is finite for all $r \in \mathbb{R}_{0}^{+}$.

The proof of the theorem is based on a couple of lemmata dealing with the special case that $d=2$ and all bodies involved are deterministic. In this special case we will give an upper bound for the volume of $(\operatorname{conv} X+r Y) \backslash(X+r Y)$. This bound will involve the minimal $Y$-distance from points of $(\operatorname{conv} X+r Y) \backslash(X+r Y)$ to conv $X$, which can be expressed using the function $w$ we are going to define now. Let $B \subseteq \mathbb{R}^{d}, d \in \mathbb{N}$, be a convex body with $0 \in \operatorname{int} B$. Then consider the function

$$
\begin{aligned}
w=w_{B}: \mathbb{R}_{0}^{+} & \rightarrow \mathbb{R}_{0}^{+}, \\
r & \mapsto \min \left\{d_{B}(y, z) \mid y \in B^{d}, z \in \mathbb{R}^{d}, y \in \Pi_{B}(0 y, z), d_{B}(0, z)=r\right\},
\end{aligned}
$$

where $x y:=\{\lambda x+(1-\lambda) y \mid \lambda \in \mathbb{R}\}$ is the line through $x$ and $y$ in case $x \neq y$ and the singleton $\{x\}$ otherwise. In the present paper we will only need the case $d=2$, but the greater generality will be helpful in the paper announced in section 5 .

Now we will show that the minimum in (6) is attained. Fix $r \in \mathbb{R}_{0}^{+}$. Choose sequences $\left(y_{k}\right)_{k \in \mathbb{N}^{+}}$in $B^{d}$ and $\left(z_{k}\right)_{k \in \mathbb{N}^{+}}$in $\mathbb{R}^{d}$ with $y_{k} \in \Pi_{B}\left(0 y_{k}, z_{k}\right)$ and $d_{B}\left(0, z_{k}\right)=r$ for all $k \in \mathbb{N}^{+}$such that $\left(d_{B}\left(y_{k}, z_{k}\right)\right)_{k \in \mathbb{N}^{+}}$converges to the infimum of the set from (6). By the compactness of $B^{d}$ and $\left\{x \in \mathbb{R}^{d} \mid d_{B}(0, x)=r\right\}$ we may assume that $\left(y_{k}\right)_{k \in \mathbb{N}^{+}}$ and $\left(z_{k}\right)_{k \in \mathbb{N}^{+}}$converge to points $y \in B^{d}$ and $z \in \mathbb{R}^{d}$ with $d_{B}(0, z)=r$. For all $k \in \mathbb{N}^{+}$ we have $d_{B}\left(y_{k}, z_{k}\right) \leq d_{B}\left(\lambda y_{k}, z_{k}\right)$ for all $\lambda \in \mathbb{R}$. Hence we have $d_{B}(y, z) \leq d_{B}(\lambda y, z)$ for all $\lambda \in \mathbb{R}$, which means $y \in \Pi_{B}(0 y, z)$.

Example 3.2. In order to get familiar with the function $w$, we compute it in the special case $B=R B^{d}, R>0$.

We will need the relation

$$
d_{B}(x, y)=\inf \left\{t \geq 0 \mid y \in x+t R B^{d}\right\}=\inf \{t \geq 0 \mid\|y-x\| \leq t R\}=\frac{\|y-x\|}{R}
$$

for $x, y \in \mathbb{R}^{d}$ several times.

Let $r \leq \frac{1}{R}$. Then choose a point $z \in \mathbb{R}^{d}$ with $\|z\|=r R$ and put $y:=z$. Then we have $\|y\|=r R \leq 1$, which implies $y \in B^{d}$. Moreover we have $d_{B}(0, z)=r$, $y \in \Pi_{B}(0 y, z)$ and $d_{B}(y, z)=0$. Hence $w_{R B^{d}}(r)=0$. 

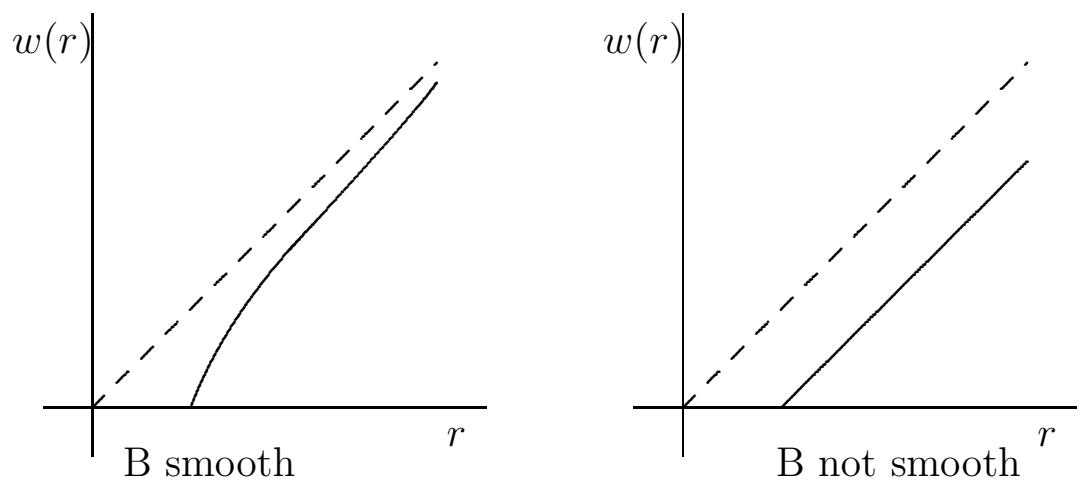

The figure on the left shows the function $w$ for a smooth gauge body $B$. The striped line is the identity. The figure on the right shows the function $w$ for a gauge body that is not smooth.

Figure 1: Typical charts of the function $w$

Now let $r \geq \frac{1}{R}$. Let $z \in \mathbb{R}^{d}$ and $y \in B^{d}$ with $d_{B}(0, z)=r$ and $y \in \Pi_{B}(0 y, z)$. The latter condition implies that the lines $0 y$ and $y z$ are perpendicular to each other. Hence we conclude:

$$
(r R)^{2}=\left(R \cdot d_{B}(0, z)\right)^{2}=\|z\|^{2}=\|y\|^{2}+\|z-y\|^{2} \leq 1+\left(R \cdot d_{B}(y, z)\right)^{2} .
$$

Thus

$$
d_{B}(y, z) \geq \sqrt{r^{2}-\left(\frac{1}{R}\right)^{2}}
$$

We have shown

$$
w_{R B^{d}}(r) \geq \sqrt{r^{2}-\left(\frac{1}{R}\right)^{2}} .
$$

Now choose $y \in B^{d}$ with $\|y\|=1$ and $z \in \mathbb{R}^{d}$ such that $\|z\|=r R$ and $y z$ is perpendicular to $0 y$. Then obviously $d_{B}(0, z)=r$ and $y \in \Pi_{B}(0 y, z)$ hold and

$$
d_{B}(y, z)=\sqrt{r^{2}-\left(\frac{1}{R}\right)^{2}}
$$

can be shown in the same way as (7).

Hence

$$
w_{R B^{d}}(r)=\sqrt{r^{2}-\left(\frac{1}{R}\right)^{2}} .
$$

Now we return to a general gauge body $B$. We will show the inequality

$$
\frac{d_{B}(y, x)}{\left\|y-x_{1}\right\|} \geq w_{B}\left(\frac{d_{B}\left(x_{1}, x\right)}{\left\|y-x_{1}\right\|}\right)
$$

for $x, x_{1}, y \in \mathbb{R}^{d}$ with $x \in \Pi_{B}\left(x_{1} x, y\right)$. Under the additional assumption $x_{1}=0$ and $\|y\|=1$ this inequality follows immediately from (6). The assumption $\|y\|=1$ can be dropped by the homogenity of $d_{B}$ and the assumption $x_{1}=0$ be the translation invariance von $d_{B}$. 
Moreover,

$$
w_{B}(r) \leq \frac{r}{s} \cdot w_{B}(s), \quad 0 \leq r \leq s
$$

holds. Indeed, there are $z \in \mathbb{R}^{d}$ and $y \in B^{d}$ satisfying $d_{B}(0, z)=s, y \in \Pi_{B}(0 y, z)$ and $d_{B}(y, z)=w(s)$. Set $z^{\prime}:=\frac{r}{s} z$ and $y^{\prime}:=\frac{r}{s} y$. Then for all $x$ in $0 y$ we have

$$
d_{B}\left(\frac{r}{s} x, z^{\prime}\right)=\frac{r}{s} \cdot d_{B}(x, z) \geq \frac{r}{s} \cdot d_{B}(y, z)=d_{B}\left(y^{\prime}, z^{\prime}\right) .
$$

Since all points from $0 y^{\prime}$ have a representation of the form $\frac{r}{s} x$ with $x \in 0 y$, this shows $y^{\prime} \in \Pi_{B}\left(0 y^{\prime}, z^{\prime}\right)$. Moreover we have $d_{B}\left(0, z^{\prime}\right)=\frac{r}{s} \cdot d_{B}(0, z)=r$ and $\left\|y^{\prime}\right\|=\frac{r}{s} \leq 1$. Hence

$$
w_{B}(r) \leq d_{B}\left(y^{\prime}, z^{\prime}\right)=\frac{r}{s} \cdot d_{B}(y, z)=\frac{r}{s} \cdot w_{B}(s) .
$$

Lemma 3.3. Let $B \subseteq \mathbb{R}^{2}$ be a convex body with $0 \in \operatorname{int} B$. Then $B$ is smooth, iff $\lim _{r \rightarrow \infty} r-w_{B}(r)=0$.

Proof. First we show that $B$ is not smooth, if not $\lim _{r \rightarrow \infty} r-w_{B}(r)=0$. So we assume that there is a sequence $\left(r_{k}\right)_{k \in \mathbb{N}^{+}}$and a number $\epsilon \in(0,1)$ such that $\lim _{k \rightarrow \infty} r_{k}=\infty$ and $r_{k}-w\left(r_{k}\right)>3 \epsilon$ for all $k \in \mathbb{N}^{+}$. Then there are sequences $\left(y_{k}\right)_{k \in \mathbb{N}^{+}}$in $B^{d}$ and $\left(z_{k}\right)_{k \in \mathbb{N}^{+}}$in $\mathbb{R}^{d}$ with $d_{B}\left(0, z_{k}\right)=r_{k}, y_{k} \in \Pi_{B}\left(0 y_{k}, z_{k}\right)$ and

$$
d_{B}\left(0, z_{k}\right)>d_{B}\left(y_{k}, z_{k}\right)+3 \epsilon
$$

for all $k \in \mathbb{N}^{+}$.

By the compactness of $B^{d}$ we can assume that $\left(y_{k}\right)_{k \in \mathbb{N}^{+}}$converges to a point $y \in B^{d}$. The triangular inequality implies $d_{B}\left(0, y_{k}\right)>3 \epsilon$ for all $k \in \mathbb{N}^{+}$and hence $y_{k} \neq 0$ for all $k \in \mathbb{N}^{+}$and $y \neq 0$. Let $u_{k}$ and $u$ denote the unit vectors perpendicular to $y_{k}$ and $y$ such that $\left(y_{k}, u_{k}\right)$ and $(y, u)$ have positive orientation. Set

$$
q_{k}:=\frac{z_{k}-y_{k}}{d_{B}\left(y_{k}, z_{k}\right)}, \quad k \in \mathbb{N}^{+} .
$$

By Lemma $2.2 q_{k}$ is a boundary point of $B$ with exterior normal vector $u_{k}$ for $k \in \mathbb{N}^{+}$.

Due to the compactness of the bd $B$ we can assume that $\left(q_{k}\right)_{k \in \mathbb{N}^{+}}$converges to a point $q \in \operatorname{bd} B$. The vector $u$ is an exterior normal vector of $B$ in $q$, since for any $p \in B$ we have $\left\langle p, u_{k}\right\rangle \leq\left\langle q_{k}, u_{k}\right\rangle$ for all $k \in \mathbb{N}^{+}$and hence $\langle p, u\rangle \leq\langle q, u\rangle$. Hence $B$ has in $q$ a supporting hyperplane parallel to $0 y$.

Now we will show that $B$ has in $q$ also a supporting hyperplane parallel to the line through 0 and $y-\epsilon q$. Assume this is not the case. Then there is $\alpha>0$ such that $p:=q+\alpha(y-\epsilon q) \in \operatorname{int} B$. Since $0 \in \operatorname{int} B$, we have $\langle q, u\rangle>0$ and hence $q$ and $y$ are a base of $\mathbb{R}^{2}$. So for all large enough $k$ the following conditions are fulfilled:

$$
\begin{gathered}
p_{k}:=q_{k}+\alpha(y-\epsilon q) \in B \\
y_{k}=\beta_{k}^{(1,1)} y+\beta_{k}^{(1,2)} q \text { and } q_{k}=\beta_{k}^{(2,1)} y+\beta_{k}^{(2,2)} q \text { with } \frac{3}{4} \leq \beta_{k}^{(1,1)} \leq \frac{5}{4}, \\
\left|\beta_{k}^{(1,2)}\right| \leq \frac{\epsilon}{4},\left|\beta_{k}^{(2,1)}\right|<\frac{1}{4} \text { and } \frac{3}{4} \leq \beta_{k}^{(2,2)} \leq \frac{5}{4}, \\
d_{B}\left(y_{k}, z_{k}\right) \cdot \frac{\alpha}{4}>1 .
\end{gathered}
$$


Now fix a number $k \in \mathbb{N}^{+}$, for which the conditions (11)-(13) are fulfilled. Then (12) implies $\beta_{k}^{(1,1)} \beta_{k}^{(2,2)}-\beta_{k}^{(2,1)} \beta_{k}^{(1,2)} \neq 0$ and using again (12) we conclude

$$
\begin{aligned}
y-\epsilon q= & (y-\epsilon q) \cdot \frac{\beta_{k}^{(1,1)} \beta_{k}^{(2,2)}-\beta_{k}^{(2,1)} \beta_{k}^{(1,2)}}{\beta_{k}^{(1,1)} \beta_{k}^{(2,2)}-\beta_{k}^{(2,1)} \beta_{k}^{(1,2)}} \\
= & \frac{\beta_{k}^{(2,2)}\left(\beta_{k}^{(1,1)} y+\beta_{k}^{(1,2)} q\right)-\beta_{k}^{(1,2)}\left(\beta_{k}^{(2,1)} y+\beta_{k}^{(2,2)} q\right)}{\beta_{k}^{(1,1)} \beta_{k}^{(2,2)}-\beta_{k}^{(2,1)} \beta_{k}^{(1,2)}} \\
& \quad-\epsilon \frac{\beta_{k}^{(1,1)}\left(\beta_{k}^{(2,1)} y+\beta_{k}^{(2,2)} q\right)-\beta_{k}^{(2,1)}\left(\beta_{k}^{(1,1)} y+\beta_{k}^{(1,2)} q\right)}{\beta_{k}^{(1,1)} \beta_{k}^{(2,2)}-\beta_{k}^{(2,1)} \beta_{k}^{(1,2)}} \\
= & \frac{\left[\beta_{k}^{(2,2)} y_{k}-\beta_{k}^{(1,2)} q_{k}\right]+\epsilon\left[\beta_{k}^{(2,1)} y_{k}-\beta_{k}^{(1,1)} q_{k}\right]}{\beta_{k}^{(1,1)} \beta_{k}^{(2,2)}-\beta_{k}^{(2,1)} \beta_{k}^{(1,2)}} \\
= & \frac{\beta_{k}^{(2,2)}+\epsilon \beta_{k}^{(2,1)}}{\beta_{k}^{(1,1)} \beta_{k}^{(2,2)}-\beta_{k}^{(2,1)} \beta_{k}^{(1,2)}}\left(y_{k}-\frac{\beta_{k}^{(1,2)}+\epsilon \beta_{k}^{(1,1)}}{\beta_{k}^{(2,2)}+\epsilon \beta_{k}^{(2,1)}} q_{k}\right) .
\end{aligned}
$$

Hence we put

$$
\tilde{\alpha}_{k}:=\alpha \cdot \frac{\beta_{k}^{(2,2)}+\epsilon \beta_{k}^{(2,1)}}{\beta_{k}^{(1,1)} \beta_{k}^{(2,2)}-\beta_{k}^{(2,1)} \beta_{k}^{(1,2)}} \quad \text { and } \quad \tilde{\epsilon}_{k}:=\frac{\beta_{k}^{(1,2)}+\epsilon \beta_{k}^{(1,1)}}{\beta_{k}^{(2,2)}+\epsilon \beta_{k}^{(2,1)}} .
$$

So we have $\tilde{\alpha}_{k}>\frac{1}{4} \alpha, \tilde{\epsilon}_{k}<3 \epsilon$ and

$$
p_{k}=q_{k}+\alpha(y-\epsilon q)=q_{k}+\tilde{\alpha}_{k}\left(y_{k}-\tilde{\epsilon}_{k} q_{k}\right) .
$$

We let $\left[p_{k}, q_{k}\right]$ denote the line segment from $p_{k}$ to $q_{k}$ and conclude

$$
\begin{aligned}
d_{B}\left(0, z_{k}\right) & =\inf \left\{t \geq 0 \mid z_{k} \in t B\right\} \\
& \leq \inf \left\{t \geq 0 \mid z_{k} \in t\left[p_{k}, q_{k}\right]\right\} \\
& =\inf \left\{t \geq 0 \mid \exists_{\lambda \in\left[0, \tilde{\alpha}_{k}\right]}: z_{k}=t \cdot\left(q_{k}+\lambda\left(y_{k}-\tilde{\epsilon}_{k} q_{k}\right)\right)\right\} \\
& =\inf \left\{t \geq 0 \mid \exists_{\lambda \in\left[0, \tilde{\alpha}_{k}\right]}: z_{k}=t \lambda y_{k}+t\left(1-\lambda \tilde{\epsilon}_{k}\right) q_{k}\right\} \\
& =\inf \left\{t \geq 0 \mid \exists_{\mu \in\left[0, t \tilde{\alpha}_{k}\right]}: z_{k}=\mu y_{k}+\left(t-\mu \tilde{\epsilon}_{k}\right) q_{k}\right\} .
\end{aligned}
$$

Now we will show that $t_{0}:=d_{B}\left(y_{k}, z_{k}\right)+\tilde{\epsilon}_{k}$ is contained in the set over which the infimum is taken in the last line. Indeed

$$
\mu_{0}:=1 \in\left[0, d_{B}\left(y_{k}, z_{k}\right) \frac{\alpha}{4}\right] \subseteq\left[0, t_{0} \tilde{\alpha}_{k}\right]
$$

because of (13) and

$$
\mu_{0} y_{k}+\left(t_{0}-\mu_{0} \tilde{\epsilon}_{k}\right) q_{k}=y_{k}+d_{B}\left(y_{k}, z_{k}\right) q_{k}=z_{k}
$$

Thus we have

$$
d_{B}\left(0, z_{k}\right) \leq d_{B}\left(y_{k}, z_{k}\right)+\tilde{\epsilon}_{k}<d_{B}\left(y_{k}, z_{k}\right)+3 \epsilon,
$$

which contradicts (10). Hence $B$ has more than one supporting hyperplane in $q$ and therefore it is not smooth. 
Now assume that $B$ is not smooth. Choose a point $q \in \mathrm{bd} B$ with unit normal vectors $u$ and $v$. Let $y \in \mathbb{R}^{2}$ denote the unit vector perpendicular to $u$ such that there is $\epsilon>0$ with $\langle y-\epsilon q, v\rangle=0$.

Let $r>0$ be a number with $y \in r B$. Then it is easy to see that there is $s>0$ such that $z=y+s q$ fulfills $d_{B}(0, z)=r$. Lemma 2.2 implies $d_{B}(y, z)=s$ and $y \in \Pi_{B}(0 y, z)$. Now we have

$$
\langle z, v\rangle=\langle y, v\rangle+s\langle q, v\rangle=\epsilon\langle q, v\rangle+s\langle q, v\rangle=(s+\epsilon) h_{B}(v) .
$$

Hence

$$
r=d_{B}(0, z) \geq \frac{\langle z, v\rangle}{h_{B}(v)}=s+\epsilon=d_{B}(y, z)+\epsilon \geq w_{B}(r)+\epsilon .
$$

So $r-w_{B}(r)$ does not converge to 0 .

A convex subset $F$ of a convex body $L$ is called face of $L$, if $\frac{1}{2}(x+y) \in F$ for two points $x, y \in L$ implies $x, y \in F$. A point $x$ is called an extreme point of a convex body $L$, if $\{x\}$ is a face of $L$. The set of all extreme points of $L$ is denoted by ext $L$.

Lemma 3.4. Let $B \subseteq \mathbb{R}^{d}$ be a convex body with $0 \in \operatorname{int} B, K \subseteq \mathbb{R}^{d}$ be an arbitrary body, $r \geq 0$ and put $L:=\operatorname{conv} K$. Let $\gamma$ be an upper bound for the length of a segment in the boundary of $L$. Then

$$
\begin{aligned}
& (L+r B) \backslash(K+r B) \subseteq \\
& \left\{x \in \mathbb{R}^{d} \mid \Pi_{B}(L, x) \cap \operatorname{ext} L=\emptyset, d_{B}(L, x) \in\left(\gamma \cdot w\left(\frac{r}{\gamma}\right), r\right]\right\} \cup(L \backslash(K+r B)) .
\end{aligned}
$$

Proof. Let $x \in(L+r B) \backslash(K+r B)$. If $x \in L$, then $x \in L \backslash(K+r B)$.

So assume $x \notin L$ from now on. Then trivially we have $d_{B}(L, x) \leq r$.

Now let $y \in \Pi_{B}(L, x)$. Since $d_{B}(y, x) \leq r<d_{B}(K, x)$ we have $y \notin K$. By $[8$, Theorem 2.1.2] there is a face $F$ of $L$ containing $y$ in its relative interior. There is a point $x_{1} \in K \cap F$, since $L \backslash F$ is convex and therefore $K \subseteq L \backslash F$ would contradict the definition of the convex hull. Since $y \notin K$, we have $x_{1} \neq y$. Hence $y$ is not extreme. So we have shown $\Pi_{B}(L, x) \cap \operatorname{ext} L=\emptyset$.

It remains to show $d_{B}(L, x)>\gamma \cdot w\left(\frac{r}{\gamma}\right)$. Choose $y \in \Pi_{B}(L, x)$. Let $F$ be the face of $L$ which contains $y$ in its relative interior and choose $x_{1} \in F \cap K$.

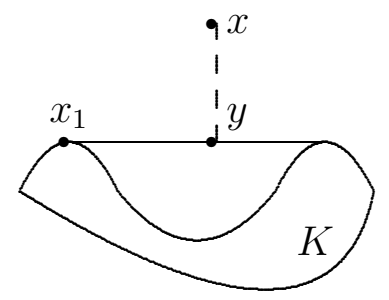

Now we want to show $y \in \Pi_{B}\left(x_{1} y, x\right)$. In other words we want to show that $y$ is the minimum of

$$
x_{1} y \rightarrow \mathbb{R}, y^{\prime} \mapsto d_{B}\left(y^{\prime}, x\right)
$$


We put $\rho:=d_{B}(y, x)$ and assume that there is $\tilde{y} \in x_{1} y$ with $d_{B}(\tilde{y}, x)<\rho$. Since $y$ is contained in the relative interior of $F$ and both $y$ and $y^{\prime}$ are in its affine hull, there is $\lambda \in(0,1)$ with $(1-\lambda) y+\lambda y^{\prime} \in F \subseteq L$. Since $x-y \in \rho B$ and $x-\tilde{y} \in \operatorname{int} \rho B$, we get

$$
x-\left((1-\lambda) y+\lambda y^{\prime}\right)=(1-\lambda)(x-y)+\lambda\left(x-y^{\prime}\right) \in \operatorname{int} \rho B
$$

and hence $d_{B}\left((1-\lambda) y+\lambda y^{\prime}, x\right)<\rho$, contradicting $y \in \Pi_{B}(L, x)$. Thus $y \in$ $\Pi_{B}\left(x_{1} y, x\right)$.

Since $d_{B}\left(x_{1}, x\right)>r$ and $\left\|y-x_{1}\right\| \leq \gamma$, the inequalities (8) and (9) imply

$$
\begin{aligned}
d_{B}(L, x) & =d_{B}(y, x) \\
& \geq\left\|y-x_{1}\right\| \cdot w\left(\frac{d_{B}\left(x_{1}, x\right)}{\left\|y-x_{1}\right\|}\right) \\
& \geq\left\|y-x_{1}\right\| \cdot \frac{d_{B}\left(x_{1}, x\right) \cdot \gamma}{\left\|y-x_{1}\right\| \cdot r} \cdot w\left(\frac{r}{\gamma}\right) \\
& >\gamma \cdot w\left(\frac{r}{\gamma}\right) .
\end{aligned}
$$

We will now provide a lemma that will be used in order to compute the volume of the right-hand side of (14). For a convex body $K \subseteq \mathbb{R}^{d}$ and $u \in \mathbb{R}^{d}$ we call the set

$$
H_{K}(u):=\left\{x \in K \mid\langle x, u\rangle=h_{K}(u)\right\}
$$

support set, where $h_{K}: \mathbb{R}^{d} \rightarrow \mathbb{R}$ is the support function. For $x, y \in \mathbb{R}^{d}, x \neq y$, we put $(x, y):=\{\lambda x+(1-\lambda) y \mid \lambda \in(0,1)\}$.

Lemma 3.5. Let $L \subseteq \mathbb{R}^{2}$ be a convex body. Let $x_{1}, x_{2} \in L, x_{1} \neq x_{2}$, be two points satisfying $\left(x_{1}, x_{2}\right) \subseteq \mathrm{bd} L$. Let $u$ denote an exterior unit normal vector of $L$ in the points in $\left(x_{1}, x_{2}\right)$ and

$$
X_{r}:=\left\{x \in \mathbb{R}^{2} \mid \Pi_{B}(L, x) \subseteq\left(x_{1}, x_{2}\right), d_{B}(L, x) \leq r,\langle x, u\rangle \geq\left\langle x_{1}, u\right\rangle\right\}, r \geq 0 .
$$

Let $q, q^{\prime} \in \mathbb{R}^{2}$ be the points satisfying $H_{B}(u)=\left[q, q^{\prime}\right]$ and, if $q \neq q^{\prime}$, then additionally $\left\langle q-q^{\prime}, x_{1}-x_{2}\right\rangle>0$. By $\Delta:=\frac{1}{2} h_{B}(u)\left\|q-q^{\prime}\right\|$ we denote the area of the triangle with vertices $0, q$ and $q^{\prime}$. Then

$$
V_{2}\left(X_{r}\right)=\left\{\begin{array}{lll}
\left\|x_{2}-x_{1}\right\| h_{B}(u) r-\Delta r^{2} & \text { if } & \left\|q-q^{\prime}\right\| r \leq\left\|x_{2}-x_{1}\right\| \\
\frac{\left\|x_{2}-x_{1}\right\|^{2} h_{B}(u)}{2\left\|q-q^{\prime}\right\|} & \text { if } & \left\|q-q^{\prime}\right\| r>\left\|x_{2}-x_{1}\right\| .
\end{array}\right.
$$

Remark 3.6. In Lemma 3.5 the vector $u$ is uniquely determined and the condition $\langle x, u\rangle \geq\left\langle x_{1}, u\right\rangle$ in the definition of $X_{r}$ is redundant, unless $L$ is contained in a line.

Remark 3.7. In Lemma 3.5 the case $q=q^{\prime}$ is more important than the case $q \neq q^{\prime}$. In the first case the assertion simplifies to

$$
V_{2}\left(X_{r}\right)=\left\|x_{2}-x_{1}\right\| h_{B}(u) r
$$


Proof of Lemma 3.5. Below we will show that $X_{r}$ is a triangle or a trapezium whose boundary consists of $\left[x_{1}, x_{2}\right]$ as well as parts of the lines $g_{1}$ parallel to $q^{\prime}$ through $x_{1}$, $g_{2}$ parallel to $q$ through $x_{2}$ and, if $\left\|q-q^{\prime}\right\| r>\left\|x_{2}-x_{1}\right\|$,

$$
g:=\left\{x \in \mathbb{R}^{2} \mid\langle x, u\rangle=\left\langle x_{1}, u\right\rangle+r h_{B}(u)\right\} .
$$

If $X_{r}$ is a triangle, then its edges are parallel to the edges of the triangle with vertices $0, q$ and $q^{\prime}$. Hence the two triangles are similar. Since the scaling factor is $\left\|x_{2}-x_{1}\right\| /\left\|q-q^{\prime}\right\|$, we get

$$
V_{2}\left(X_{r}\right)=\left(\frac{\left\|x_{2}-x_{1}\right\|}{\left\|q-q^{\prime}\right\|}\right)^{2} \cdot \Delta=\frac{\left\|x_{2}-x_{1}\right\|^{2} h_{B}(u)}{2\left\|q-q^{\prime}\right\|} .
$$

If $X_{r}$ is a trapezium, then denote by $Z_{r}$ the triangle bounded by $g$ and the lines parallel to $q$ resp. $q^{\prime}$ through $x_{2}$. Observing that $X_{r} \cap Z_{r}$ is contained in a line and $X_{r} \cup Z_{r}$ is a parallelogram of side length $\left\|x_{2}-x_{1}\right\|$ and height $r h_{B}(u)$, one gets

$$
V_{2}\left(X_{r}\right)=V_{2}\left(X_{r} \cup Z_{r}\right)-V_{2}\left(Z_{r}\right)=\left\|x_{2}-x_{1}\right\| h_{B}(u) r-\Delta r^{2} .
$$

Now we prove that $X_{r}$ is indeed bounded by the segments mentioned above.

It is clear that $X_{r}$ is bounded by $\left[x_{1}, x_{2}\right]$.

In order to show that $X_{r}$ is bounded by parts of $g_{1}$ and $g_{2}$, we first show that the equivalence

$$
\Pi_{B}(L, x) \subseteq\left(x_{1}, x_{2}\right) \Longleftrightarrow \Pi_{B}\left(x_{1} x_{2}, x\right) \subseteq\left(x_{1}, x_{2}\right)
$$

holds for $x \in \mathbb{R}^{2}$ with $\langle x, u\rangle \geq\left\langle x_{1}, u\right\rangle$. The implication " $\Leftarrow$ " follows from the fact that for any $y \in L \backslash x_{1} x_{2}$ there is $y^{\prime} \in x_{1} x_{2}$ with $d_{B}\left(y^{\prime}, x\right)<d_{B}(y, x)$. Now assume $\Pi_{B}(L, x) \subseteq\left(x_{1}, x_{2}\right)$. The set $I:=x_{1} x_{2} \cap\left(x+d_{B}(L, x) B^{*}\right)$, where $B^{*}:=\{-p \mid$ $p \in B\}$, is convex and contains at least one point of $\left(x_{1}, x_{2}\right)$, but neither $x_{1}$ nor $x_{2}$. Hence $I \subseteq\left(x_{1}, x_{2}\right)$ and therefore $\Pi_{B}\left(x_{1} x_{2}, x\right) \subseteq\left(x_{1}, x_{2}\right)$.

By the equality $\Pi_{B}\left(x_{1} x_{2}, x\right)=x-d_{B}\left(x_{1} x_{2}, x\right)\left[q, q^{\prime}\right]$, from Lemma 2.2 , if $x \notin x_{1} x_{2}$, and is trivial otherwise, we have

$$
\Pi_{B}(L, x) \subseteq\left(x_{1}, x_{2}\right) \Longleftrightarrow x-d_{B}\left(x_{1} x_{2}, x\right)\left[q, q^{\prime}\right] \subseteq\left(x_{1}, x_{2}\right)
$$

and the condition on the right-hand side is equivalent to $x$ lying between $g_{1}$ and $g_{2}$.

In order to show that a segment of $g$ bounds $X_{r}$, iff $\left\|q-q^{\prime}\right\| r \leq\left\|x_{2}-x_{1}\right\|$, we prove

$$
d_{B}\left(x_{1} x_{2}, x\right) \leq r \Longleftrightarrow\langle x, u\rangle \leq\left\langle x_{1}, u\right\rangle+r h_{B}(u)
$$

for any point $x \in \mathbb{R}^{2}$ with $\langle x, u\rangle \geq\left\langle x_{1}, u\right\rangle$. Assume $d_{B}\left(x_{1} x_{2}, x\right) \leq r$. Then there is $y \in x_{1} x_{2}$ and $b \in B$ with $x=y+r b$ and hence

$$
\langle x, u\rangle=\langle y, u\rangle+r\langle b, u\rangle \leq\left\langle x_{1}, u\right\rangle+r h_{B}(u) .
$$

Now assume $\langle x, u\rangle=\left\langle x_{1}, u\right\rangle+s h_{B}(u)$ for $s \leq r$. Put $y:=x-s q$. Then $y \in x_{1} x_{2}$ and $\frac{s}{r} q \in B$, which implies

$$
x=y+r \frac{s}{r} q \in x_{1} x_{2}+r B
$$


and hence $d_{B}\left(x_{1} x_{2}, x\right) \leq r$.

For $x \in \mathbb{R}^{2}$ with $\langle x, u\rangle \geq\left\langle x_{1}, u\right\rangle$ and $\Pi_{B}(L, x) \subseteq\left(x_{1}, x_{2}\right)$ we have trivially $\Pi_{B}(L, x) \subseteq x_{1} x_{2}$ and, as shown above, $\Pi_{B}\left(x_{1} x_{2}, x\right) \subseteq\left(x_{1}, x_{2}\right) \subseteq L$. Hence $d_{B}(L, x)=$ $d_{B}\left(x_{1} x_{2}, x\right)$ and thus

$$
d_{B}(L, x) \leq r \Longleftrightarrow\langle x, u\rangle \leq\left\langle x_{1}, u\right\rangle+r h_{B}(u)
$$

which shows that $X_{r}$ is bounded by a segment of $g$, iff $g$ intersects the triangle bounded by $x_{1} x_{2}, g_{1}$ and $g_{2}$. This is obviously the case, iff $\left\|q-q^{\prime}\right\| r \leq\left\|x_{2}-x_{1}\right\|$.

Lemma 3.8. Let $B \subseteq \mathbb{R}^{2}$ be a convex body with $0 \in \operatorname{int} B, K \subseteq \mathbb{R}^{2}$ be an arbitrary body and $\gamma$ an upper bound for the length of a segment in the boundary of $L:=$ conv $K$. Then

$$
V_{2}((L+r B) \backslash(K+r B)) \leq V_{2}((L+B) \backslash L) \cdot \gamma \cdot\left(\frac{r}{\gamma}-w_{B}\left(\frac{r}{\gamma}\right)\right)+V_{2}(L \backslash(K+r B)) .
$$

Proof. Denote by $S$ the set of all singular unit normal vectors of $L$, i.e. the set of all unit normal vectors of $L$ in points in the relative interior of segments in the boundary of $L$. By [8, Theorem 2.2.5] the set $S$ is at most countable. We have $(\operatorname{bd} L) \backslash(\operatorname{ext} L) \subseteq \bigcup_{u \in S} H_{L}(u)$, since by [8, Theorem 2.1.2] every boundary point of $L$ is contained in a 0 - or 1 -dimensional face.

For a point $x \in \mathbb{R}^{2} \backslash L$ the set $\Pi_{B}(L, x)$ is convex and does not intersect the interior of $L$. So, if $\Pi_{B}(L, x) \cap(\operatorname{ext} L)=\emptyset$ and hence $\Pi_{B}(L, x) \subseteq(\operatorname{bd} L) \backslash(\operatorname{ext} L)$, then there is $u \in S$ with $\Pi_{B}(L, x) \subseteq H_{L}(u)$. Hence Lemma 3.4 yields

$$
\begin{gathered}
(L+r B) \backslash(K+r B) \\
\subseteq \bigcup_{u \in S}\left\{x \in \mathbb{R}^{2} \mid \Pi_{B}(L, x) \subseteq H_{L}(u), d_{B}(L, x) \in\left(\gamma \cdot w\left(\frac{r}{\gamma}\right), r\right],\right. \\
\left.\langle x, u\rangle \geq h_{L}(u)\right\} \cup(L \backslash(K+r B)) .
\end{gathered}
$$

We put $s:=\gamma \cdot w_{B}\left(\frac{r}{\gamma}\right)$ and

$$
T_{\rho, u}:=\left\{x \in \mathbb{R}^{2} \backslash L \mid \Pi_{B}(L, x) \subseteq H_{L}(u), d_{B}(L, x) \leq \rho,\langle x, u\rangle \geq h_{L}(u)\right\}
$$

for $\rho>0$ and $u \in S$. Then we have

$$
V_{2}((L+r B) \backslash(K+r B)) \leq \sum_{u \in S} V_{2}\left(T_{r, u} \backslash T_{s, u}\right)+V_{2}(L \backslash(K+r B)) .
$$

For $u \in S$ denote the length of $H_{L}(u)$ by $\lambda_{u}$ and the length of $H_{B}(u)$ by $\beta_{u}$. Let $u \in S$. In order to derive

$$
V_{2}\left(T_{r, u} \backslash T_{s, u}\right) \leq \lambda_{u} h_{B}(u)(r-s)
$$

from Lemma 3.5 we have to distinguish three cases. First assume $\beta_{u} \cdot r \leq \lambda_{u}$. Then we have $\beta_{u} \cdot s \leq \lambda_{u}$ and hence

$$
V_{2}\left(T_{r, u} \backslash T_{s, u}\right)=\lambda_{u} h_{B}(u) r-c r^{2}-\lambda_{u} h_{B}(u) s+c s^{2} \leq \lambda_{u} h_{B}(u)(r-s),
$$

where $c:=\frac{1}{2} \beta_{u} h_{B}(u) \geq 0$. 
Now assume $\beta_{u} \cdot r>\lambda_{u}$, but $\beta_{u} \cdot s \leq \lambda_{u}$. Then

$$
\begin{aligned}
V_{2}\left(T_{r, u} \backslash T_{s, u}\right) & =\frac{\left(\lambda_{u}\right)^{2} h_{B}(u)}{2 \beta_{u}}-\lambda_{u} h_{B}(u) s+\frac{1}{2} \beta_{u} h_{B}(u) s^{2} \\
& \leq \frac{1}{2} \lambda_{u} h_{B}(u) r-\lambda_{u} h_{B}(u) s+\frac{1}{2} \lambda_{u} h_{B}(u) s \\
& \leq \lambda_{u} h_{B}(u)(r-s) .
\end{aligned}
$$

Finally assume $\beta_{u} \cdot r>\lambda_{u}$ and $\beta_{u} \cdot s>\lambda_{u}$. Then

$$
V_{2}\left(T_{r, u} \backslash T_{s, u}\right)=\frac{\left(\lambda_{u}\right)^{2} h_{B}(u)}{2 \beta_{u}}-\frac{\left(\lambda_{u}\right)^{2} h_{B}(u)}{2 \beta_{u}}=0 \leq \lambda_{u} h_{B}(u)(r-s) .
$$

Hence we get

$$
\begin{aligned}
V_{2}((L+r B) \backslash(K+r B)) & \leq \sum_{u \in S}\left(\lambda_{u} h_{B}(u) \cdot(r-s)\right)+V_{2}(L \backslash(K+r B)) \\
& \leq\left(\sum_{u \in S} \lambda_{u} h_{B}(u)\right) \cdot\left(r-\gamma \cdot w_{B}\left(\frac{r}{\gamma}\right)\right)+V_{2}(L \backslash(K+r B)) \\
& \leq V_{2}((L+B) \backslash L) \cdot \gamma \cdot\left(\frac{r}{\gamma}-w_{B}\left(\frac{r}{\gamma}\right)\right)+V_{2}(L \backslash(K+r B)) .
\end{aligned}
$$

In order to justify the last inequality we choose for each $u \in S$ a vector $b_{u} \in$ bd $B$ with exterior normal vector $u$ and put $T_{u}^{\prime}:=\left\{y+\lambda b_{u} \mid y \in \operatorname{relint} H_{L}(u), \lambda \in(0,1)\right\}$, where relint $K$ denotes the relative interior of a convex body $K$. Since for $x \in T_{u}^{\prime}$ we have $\Pi_{B}(L, x) \subseteq H_{L}(u)$ and $\Pi_{B}(L, x) \cap$ relint $H_{L}(u) \neq \emptyset$ the sets $T_{u}^{\prime}, u \in S$, are pairwise disjoint. Since they are subsets of $(L+B) \backslash L$, we get

$$
\sum_{u \in S} \lambda_{u} h_{B}(u)=\sum_{u \in S} V_{2}\left(T_{u}^{\prime}\right) \leq V_{2}((L+B) \backslash L)
$$

Our last step in the preparation of the proof of Theorem 3.1 is a remark establishing a link between the function $w_{B}$ and the number $\rho_{B}$.

Remark 3.9. Let $B \subseteq \mathbb{R}^{d}$ be a convex body such that a largest ball contained in $B$ has its center at the origin. For any number $r \in \mathbb{R}_{0}^{+}$there are $y \in B^{d}$ and $z \in \mathbb{R}^{d}$ such that $d_{B}(0, z)=r, y \in \Pi_{B}(0 y, z)$ and $d_{B}(y, z)=w_{B}(r)$. Thus

$$
\begin{aligned}
r-w_{B}(r) & =d_{B}(0, z)-d_{B}(y, z) \\
& \leq d_{B}(0, y) \\
& =\inf \{t \geq 0 \mid y \in 0+t B\} \\
& \leq \inf \left\{t \geq 0 \mid y \in t \rho_{B} B^{d}\right\} \\
& =1 / \rho_{B} .
\end{aligned}
$$

Proof of Theorem 3.1. Put $X^{x}:=X \cap(x+\hat{Y})$ and $Z^{x}:=\operatorname{conv} X^{x}$ for $x \in Y^{\perp}$. Then

$$
\lim _{r \rightarrow \infty} \mathbb{E} V_{n}\left(X_{Y}+r Y\right)-\mathbb{E} V_{n}(X+r Y)=\lim _{r \rightarrow \infty} \mathbb{E} \int_{Y^{\perp}} V_{2}\left(\left(Z^{x}+r Y\right) \backslash\left(X^{x}+r Y\right)\right) d x .
$$


Assume that $X$ and $Y$ are defined on some probability space $(\Omega, \mathcal{A}, \mathbb{P})$ and fix $\omega \in \Omega$, $x \in Y^{\perp}$ and $r \geq 0$. Let $y$ denote the midpoint of a ball of maximal radius contained in $Y$. We identify $x+\hat{Y}$ and the linear subspace parallel to it with $\mathbb{R}^{2}$ at the same time. Since $(\operatorname{diam} X)$ is an upper bound for length of a segment in the boundary of $Z^{x}$, we get from Lemma 3.8

$$
\begin{aligned}
& V_{2}\left(\left(Z^{x}+r Y\right) \backslash\left(X^{x}+r Y\right)\right)=V_{2}\left(\left(Z^{x}+r(Y-y)\right) \backslash\left(X^{x}+r(Y-y)\right)\right) \\
& \leq V_{2}\left(\left(Z^{x}+(Y-y)\right) \backslash Z^{x}\right) \cdot(\operatorname{diam} X) \cdot\left(\frac{r}{\operatorname{diam} X}-w_{Y-y}\left(\frac{r}{\operatorname{diam} X}\right)\right) \\
&+V_{2}\left(Z^{x} \backslash\left(X^{x}+r(Y-y)\right)\right) .
\end{aligned}
$$

The map $\mathcal{K}_{00} \times \mathbb{R}_{0}^{+},(B, r) \mapsto w_{B}(r)$, where $\mathcal{K}_{00}$ denotes the set of all convex bodies having 0 as an interior point, will be proven to be measurable in the appendix. Now Lemma 3.3 together with the dominated convergence theorem, on which we will comment below, gives

$$
\begin{aligned}
& \lim _{r \rightarrow \infty} \mathbb{E} V_{n}\left(X_{Y}+r Y\right)-\mathbb{E} V_{n}(X+r Y) \\
& \leq \lim _{r \rightarrow \infty} \mathbb{E}\left[\int_{Y^{\perp}} V_{2}\left(\left(Z^{x}+(Y-y)\right) \backslash Z^{x}\right) \cdot(\operatorname{diam} X) \cdot\left(\frac{r}{\operatorname{diam} X}-w_{Y-y}\left(\frac{r}{\operatorname{diam} X}\right)\right)\right. \\
&\left.\quad+V_{2}\left(Z^{x} \backslash\left(X^{x}+r(Y-y)\right)\right) d x\right] \\
& \leq \lim _{r \rightarrow \infty} \mathbb{E}[ V_{n}\left(\left(X_{Y}+(Y-y)\right) \backslash X_{Y}\right) \cdot(\operatorname{diam} X) \cdot\left(\frac{r}{\operatorname{diam} X}-w_{Y-y}\left(\frac{r}{\operatorname{diam} X}\right)\right) \\
&\left.\quad+V_{n}\left(X_{Y} \backslash(X+r(Y-y))\right)\right] \\
&=0 .
\end{aligned}
$$

We have been allow to apply the dominated convergence theorem, since

$$
\frac{r}{\operatorname{diam} X}-w_{Y-y}\left(\frac{r}{\operatorname{diam} X}\right) \leq 1 / \rho_{Y-y}=1 / \rho_{Y}
$$

holds by Remark 3.9 and we assumed $\mathbb{E}\left[\left(V_{n}\left(X_{Y}+Y\right)-V_{n}\left(X_{Y}\right)\right) \cdot \operatorname{diam} X / \rho_{Y}\right]<\infty$ and $\mathbb{E} V_{n}\left(X_{Y}\right)<\infty$.

Now we show that the smoothness assumption in Theorem 3.1 can not be relaxed. More precisely, we show:

Corollary 3.10. Let $B \subseteq \mathbb{R}^{2}$ be a convex body. Then $B$ is smooth, iff

$$
\lim _{r \rightarrow \infty} V_{2}(\operatorname{conv} K+r B)-V_{2}(K+r B)=0
$$

for every body $K \subseteq \mathbb{R}^{2}$.

Proof. The "only if"-direction is an immediate consequence of Theorem 3.1.

So now assume that $B$ is not smooth. Then choose a point $q \in B$ having two exterior unit normal vectors $u_{1}, u_{3} \in \mathbb{R}^{2}, u_{1} \neq u_{3}$. We may assume $u_{1} \neq-u_{3}$. In fact, $u_{1}=-u_{3}$ implies that $B$ is a line segment, and then we can choose a new point $q$ to be one of its endpoints and new vectors $u_{1}$ and $u_{3}$ such that $u_{1} \neq-u_{3}$. Now set

$$
u_{2}:=\frac{u_{1}+u_{3}}{\left\|u_{1}+u_{3}\right\|} .
$$




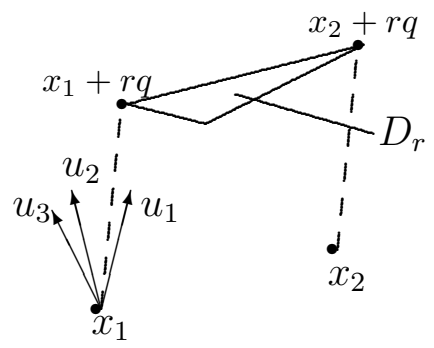

Choose points $x_{1}, x_{2} \in \mathbb{R}^{2}$ such that $x_{1}-x_{2}$ has length 1 and is perpendicular to $u_{2}$ and put $K:=\left\{x_{1}, x_{2}\right\}$. We may assume w.l.o.g. that $\left\langle x_{2}-x_{1}, u_{1}-u_{3}\right\rangle>0$.

For every $r \in \mathbb{R}^{+}$we let $D_{r}$ denote the triangle bounded by $\left[x_{1}+r q, x_{2}+r q\right]$, the line through $x_{1}+r q$ perpendicular to $u_{1}$ and the line through $x_{2}+r q$ perpendicular to $u_{3}$. Obviously $V_{2}\left(D_{r}\right)$ is constant and hence does not converge to zero. So it suffices to show

$$
\operatorname{int} D_{r} \subseteq(\operatorname{conv} K+r B) \backslash(K+r B)
$$

for all large enough $r$. For all $r \geq 0$ we have $x_{1}+r q \in K+r B$ and $x_{2}+r q \in K+r B$ and for all large enough $r$ the third corner of $D_{r}$ lies in conv $K+r B$ as well, which implies $D_{r} \subseteq$ conv $K+r B$. On the other hand, assume that there is some $z \in\left(\right.$ int $\left.D_{r}\right) \cap(K+r B)$. Then there is $p \in B$ with $z=x_{1}+r p$ or $z=x_{2}+r p$, w.l.o.g. $z=x_{1}+r p$. Now

$$
\left\langle z, u_{1}\right\rangle=\left\langle x_{1}, u_{1}\right\rangle+r\left\langle p, u_{1}\right\rangle \leq\left\langle x_{1}, u_{1}\right\rangle+r\left\langle q, u_{1}\right\rangle=\left\langle x_{1}+r q, u_{1}\right\rangle,
$$

which contradicts $z \in \operatorname{int} D_{r}$.

\section{Polynomial parallel volume and convexity}

Heveling, Hug and Last [2] proved that a body in the Euclidean plane is convex, iff its parallel volume is a polynomial. This result was generalized by Hug, Last and Weil [3] to more general 2-dimensional Minkowski spaces and to random sets. Moreover they gave a interpretation of the result for higher-dimensional bodies.

We will now give a new proof of this theorem under assumptions similar those of made in [3]. Then we will discuss applications to the Wills functional and its generalisation introduced in [4], namely the functionals $f_{\mu}$.

Recall the notation introduced at the beginning of section 3.

Theorem 4.1. Let $X \subseteq \mathbb{R}^{n}$ be a random body and $Y \subseteq \mathbb{R}^{n}$ a random disc body such that $\mathbb{E} V_{n}\left(X_{Y}+Y\right)<\infty$ and

$$
\mathbb{E}\left[\left(V_{n}\left(X_{Y}+Y\right)-V_{n}\left(X_{Y}\right)\right) \cdot \operatorname{diam} X / \rho_{Y}\right]<\infty .
$$

Then $X \cap(x+\hat{Y})$ is a.s. convex for $V_{n-2}$-almost all $x \in Y^{\perp}$, iff $\mathbb{E} V_{n}(X+r Y)$ is a polynomial. 
Proof. The "only if"-part is an immediate consequence of Lemma 2.1.

So assume that $\mathbb{E} V_{n}(X+r Y)$ is a polynomial. By the easy direction $\mathbb{E} V_{n}\left(X_{Y}+r Y\right)$ is a polynomial, too. By Theorem 3.1 the difference between these two polynomials converges to zero and thus is zero for all $r$. Therefore $V_{n}\left(X_{Y}+r Y\right)-V_{n}(X+r Y)=0$ a.s. for every $r \geq 0$. Hence for $\mathbb{P}$-a.e. $\omega \in \Omega$ and for $V_{n-2}$-a.e. $x \in Y^{\perp}$ we have

$$
V_{2}\left((\operatorname{conv} X \cap(x+\hat{Y}))+\frac{1}{k} Y\right)-V_{2}\left((X \cap(x+\hat{Y}))+\frac{1}{k} Y\right)=0, k \in \mathbb{N}^{+} .
$$

Fix such $\omega$ and $x$. As $(\operatorname{conv} X \cap(x+\hat{Y}))+\frac{1}{k} Y$ is the closure of its interior (w.r.t. the topological space $x+\hat{Y})$, it follows that

$$
(X \cap(x+\hat{Y}))+\frac{1}{k} Y=(\operatorname{conv} X \cap(x+\hat{Y}))+\frac{1}{k} Y, k \in \mathbb{N}^{+} .
$$

Now let $x_{1}, x_{2} \in X \cap(x+\hat{Y})$ and $x \in\left[x_{1}, x_{2}\right]$. Then $x \in X+\frac{1}{k} Y$ for every $k \in \mathbb{N}^{+}$, since these sets are convex, and hence $x \in X \cap(x+\hat{Y})$. So $X \cap(x+\hat{Y})$ is convex.

A signed measure is a finite measure that may take negative values. For a more formal introduction see e.g. [1, chapter 4].

Recall that we consider $\mathcal{K}$ always with the Fell-Matheron- $\sigma$-Algebra. For a signed measure $\mu$ on $\mathcal{K}$ satisfying

$$
\int_{\mathcal{K}} V_{n}(K+A) d|\mu|(A)<\infty, \quad K \in \mathcal{C}
$$

we put

$$
f_{\mu}: \mathcal{C} \rightarrow \mathbb{R}, K \mapsto \int_{\mathcal{K}} V_{n}(K+A) d \mu(A)
$$

By the results mentioned before Theorem 3.1 the map $\mathcal{C} \times \mathcal{K} \rightarrow \mathbb{R}_{0}^{+},(K, A) \mapsto$ $V_{n}(K+A)$ is measurable. Hence Fubini's theorem implies that $f_{\mu}: \mathcal{C} \rightarrow \mathbb{R}$ is measurable.

Proposition 4.2. Let $\mu$ be a signed measure on $\mathcal{K}$ and $X \subseteq \mathbb{R}^{n}$ a random convex body with $\mathbb{E} f_{|\mu|}(X)<\infty$. Then

$$
\mathbb{R}_{0}^{+} \rightarrow \mathbb{R}_{0}^{+}, r \mapsto \mathbb{E} f_{\mu}(r X)
$$

is a polynomial.

Proof. For $j=0, \ldots, n$ we have

$$
\mathbb{E} \int_{\mathcal{K}} V(X[j], A[n-j]) d|\mu|(A) \leq \mathbb{E} \int_{\mathcal{K}} V_{n}(X+A) d|\mu|(A)<\infty .
$$

Let $r \in \mathbb{R}_{0}^{+}$. Then

$$
\begin{aligned}
\mathbb{E} f_{\mu}(r X) & =\mathbb{E} \int_{\mathcal{K}} \sum_{j=0}^{n}\left(\begin{array}{l}
n \\
j
\end{array}\right) V(r X[j], A[n-j]) d \mu(A) \\
& =\sum_{j=0}^{n}\left(\begin{array}{l}
n \\
j
\end{array}\right) \mathbb{E} \int_{\mathcal{K}} V(X[j], A[n-j]) d \mu(A) \cdot r^{j} .
\end{aligned}
$$


We will now prove some theorems that give sufficient conditions for the reverse of Proposition 4.2 to hold.

Theorem 4.3. Let $\mu$ be a (non-negative) measure with (15) which is concentrated on the set of all disc bodies. Assume

$$
\mathbb{E} \int_{\mathcal{K}} V_{n}\left(X_{A}+A\right) d \mu(A)<\infty
$$

and

$$
\mathbb{E} \int_{\mathcal{K}}\left(V_{n}\left(X_{A}+A\right)-V_{n}\left(X_{A}\right)\right) \cdot(\operatorname{diam} X) / \rho_{A} d \mu(A)<\infty
$$

If now

$$
\mathbb{R}_{0}^{+} \rightarrow \mathbb{R}_{0}^{+}, r \mapsto \mathbb{E} f_{\mu}(r X)
$$

is a polynomial, then $X \cap(x+\hat{A})$ is a.s. convex for $\mu$-almost every $A$ and $V_{n-2}$-almost every $x \in A^{\perp}$.

Proof. It is easy to see that $\mathbb{E} \int_{\mathcal{K}} V_{n}\left(X_{A}+A\right) d \mu(A)<\infty$ implies $\mathbb{E} f_{\mu}(r X)<\infty$ for all $r \in \mathbb{R}_{0}^{+}$.

The measure $\tilde{\mu}:=\frac{\mu}{m}$, where $m:=\mu(\mathcal{K})$, is a probability measure. Let $Y$ be a random variable independent of $X$ and distributed according to $\tilde{\mu}$. We have assumed that there are $c_{0}, \ldots, c_{n} \in \mathbb{R}$ such that for all $r \in \mathbb{R}_{0}^{+}$we have

$$
\begin{aligned}
\sum_{j=0}^{n} c_{j} r^{j} & =\mathbb{E} f_{\mu}(r X) \\
& =r^{n} \mathbb{E} \int_{\mathcal{K}} V_{n}\left(X+\frac{1}{r} A\right) d \mu(A) \\
& =r^{n} m \mathbb{E} V_{n}\left(X+\frac{1}{r} Y\right)
\end{aligned}
$$

Dividing by $r^{n} m$ and putting $s:=\frac{1}{r}$ we obtain

$$
\sum_{j=0}^{n} \frac{c_{j}}{m} s^{n-j}=\mathbb{E} V_{n}(X+s Y) .
$$

Now Theorem 4.1 yields the assertion.

An immediate consequence of Theorem 4.3 is the following corollary:

Corollary 4.4. Let $X \subseteq \mathbb{R}^{2}$ be a random compact set and $\mu$ a measure satisfying (15), which is concentrated on the set of all disc bodies and which is not the zero measure. We assume

$$
\mathbb{E} f_{\mu}(\operatorname{conv} X)<\infty
$$

and

$$
\mathbb{E} \int_{\mathcal{K}}\left(V_{2}(\operatorname{conv} X+A)-V_{2}(\operatorname{conv} X)\right) \cdot(\operatorname{diam} X) / \rho_{A} d \mu(A)<\infty
$$

If now

$$
\mathbb{R}_{0}^{+} \rightarrow \mathbb{R}_{0}^{+}, r \mapsto \mathbb{E} f_{\mu}(r X)
$$

is a polynomial, then $X$ is a.s. convex. 
The most important functional $f_{\mu}$ is the Wills functional

$$
W: \mathcal{C} \rightarrow \mathbb{R}, K \mapsto \mathbb{E}\left[V_{n}\left(K+\Lambda B^{n}\right)\right],
$$

where $\Lambda$ is a random variable in $\mathbb{R}_{0}^{+}$with distribution function $1-e^{-\pi t^{2}}$. It is wellknown that the Wills functional of a convex body equals the sum of its intrinsic volumes.

Corollary 4.5. Let $X \subseteq \mathbb{R}^{2}$ be a random compact set with $\mathbb{E}\left[(\operatorname{diam} X)^{2}\right]<\infty$. If

$$
\mathbb{R}_{0}^{+} \rightarrow \mathbb{R}_{0}^{+}, r \mapsto \mathbb{E} W(r X)
$$

is a polynomial, then $X$ is a.s. convex.

Proof. The assertion is an immediate consequence of Corollary 4.4, if its assumptions are fulfilled. In order to check this, let $\Lambda$ be a random variable in $\mathbb{R}_{0}^{+}$with distribution function $1-e^{-\pi t^{2}}$, which is independent of $X$. Then

$$
\begin{aligned}
\mathbb{E} W(\operatorname{conv} X) & =\mathbb{E} V_{2}\left(\operatorname{conv} X+\Lambda B^{2}\right) \\
& \leq \mathbb{E} V_{2}\left((\operatorname{diam} X+\Lambda) B^{2}\right)=\pi \cdot \mathbb{E}(\operatorname{diam} X+\Lambda)^{2}<\infty
\end{aligned}
$$

and

$$
\begin{aligned}
\mathbb{E}\left(V_{2}\left(\operatorname{conv} X+\Lambda B^{2}\right)-V_{2}(\operatorname{conv} X)\right) \cdot \operatorname{diam} X / \rho_{\Lambda B^{2}} \\
\quad=\mathbb{E}\left(\sum_{j=0}^{2} \kappa_{2-j} \Lambda^{2-j} V_{j}(\operatorname{conv} X)-V_{2}(\operatorname{conv} X)\right) \cdot \operatorname{diam} X \cdot \frac{1}{\Lambda} \\
\quad=\mathbb{E}\left(\pi \Lambda^{2}+2 \Lambda V_{1}(\operatorname{conv} X)\right) \cdot \operatorname{diam} X \cdot \frac{1}{\Lambda} \\
\quad \leq \mathbb{E}(\pi \Lambda+2 \pi \operatorname{diam} X) \cdot \operatorname{diam} X \\
\quad<\infty .
\end{aligned}
$$

Thus the assumptions of Corollary 4.4 are fulfilled.

\section{Random unions of random bodies}

In this section we want discuss the application of the theorems in sections 3 and 4 to unions of a random number of random bodies. First we will present a theorem that can be used in order to check the integrability conditions of these theorems. Then we will proof that a union of randomly many random bodies in the plane which has polynomial expected parallel volume and fulfills certain independence conditions consists a.s. only of one body (which then is convex by Theorem 4.1). We do this by showing that if such a union is convex a.s., then it can only consist of one body.

Theorem 5.1. Let $B \subseteq \mathbb{R}^{n}$ be a disc body. Let $N$ be an $\mathbb{N}^{+}$-valued random variable with $\mathbb{E} N<\infty,\left(X_{i}\right)_{i \in \mathbb{N}^{+}}$a sequence of random points in $\mathbb{R}^{n}$ and $\left(Z_{i}\right)_{i \in \mathbb{N}^{+}}$a sequence of random bodies in $\mathbb{R}^{n}$ with $0 \in Z_{i}$ a.s. for all $i \in \mathbb{N}^{+}$. Assume that $N$ is independent of $\left(\left(X_{i}, Z_{i}\right)\right)_{i \in \mathbb{N}^{+}}$. Put $Z:=\bigcup_{i=1}^{N}\left(X_{i}+Z_{i}\right)$. If there are numbers $S_{1}$ and $S_{2}$ such that $\mathbb{E}\left(\operatorname{diam} Z_{i}\right)^{n}<S_{1}$ and $\mathbb{E}\left\|X_{i}\right\|^{n}<S_{2}$ for all $i \in \mathbb{N}^{+}$, then

$$
\mathbb{E} V_{n}\left(Z_{B}+B\right)<\infty \quad \text { and } \quad \mathbb{E}\left[\left(V_{n}\left(Z_{B}+B\right)-V_{n}\left(Z_{B}\right)\right) \cdot(\operatorname{diam} Z) / \rho_{B}\right]<\infty .
$$


The proof of the theorem is based on the following lemma:

Lemma 5.2. Let $N$ be an $\mathbb{N}^{+}$-valued random variable with $\mathbb{E} N<\infty$ and $\left(T_{i}\right)_{i \in \mathbb{N}^{+}}$a sequence of $\mathbb{R}_{0}^{+}$-valued random variables which is independent of $N$, such that there is a number $S$ with $\mathbb{E} T_{i}^{j}<S$. Then

$$
\mathbb{E} \max \left\{T_{i} \mid i=1, \ldots, N\right\}^{j}<\infty .
$$

Proof. We have

$$
\begin{aligned}
\mathbb{E} \max \left\{T_{i} \mid i=1, \ldots, N\right\}^{j} & =\sum_{k=1}^{\infty} \mathbb{P}(N=k) \cdot \mathbb{E} \max \left\{T_{i} \mid i=1, \ldots, k\right\}^{j} \\
& \leq \sum_{k=1}^{\infty} \mathbb{P}(N=k) \cdot \mathbb{E}\left[T_{1}^{j}+\cdots+T_{k}^{j}\right] \\
& \leq \sum_{k=1}^{\infty} \mathbb{P}(N=k) \cdot k S \\
& =S \cdot \mathbb{E} N \\
& <\infty
\end{aligned}
$$

Proof of Theorem 5.1. We have a.s.

$$
\begin{aligned}
(\operatorname{diam} Z)^{n} & \leq\left(\max _{i, k \in\{1, \ldots, N\}} \operatorname{diam} Z_{i}+\left\|X_{i}\right\|+\left\|X_{k}\right\|+\operatorname{diam} Z_{k}\right)^{n} \\
& \leq\left(4 \max \left\{\left\|X_{i}\right\|, \operatorname{diam} Z_{i} \mid i=1, \ldots, N\right\}\right)^{n} \\
& \leq 4^{n} \max \left\{\left\|X_{i}\right\| \mid i=1, \ldots, N\right\}^{n}+4^{n} \max \left\{\operatorname{diam} Z_{i} \mid i=1, \ldots, N\right\}^{n} .
\end{aligned}
$$

Since

$$
\mathbb{E} \max \left\{\left\|X_{i}\right\| \mid i=1, \ldots, N\right\}^{n}<\infty
$$

and $\mathbb{E} \max \left\{\operatorname{diam} Z_{i} \mid i=1, \ldots, N\right\}^{n}<\infty$

by Lemma 5.2 , this gives $\mathbb{E}(\operatorname{diam} Z)^{n}<\infty$. As we have

$$
V_{n}\left(Z_{B}+B\right) \leq V_{n}\left((\operatorname{diam} Z+\operatorname{diam} B) B^{n}\right)=\kappa_{n}(\operatorname{diam} Z+\operatorname{diam} B)^{n}
$$

and, in the notation of the proof of Theorem 3.1,

$$
\begin{aligned}
V_{n}\left(Z_{B}+B\right)-V_{n}\left(Z_{B}\right) & =\int_{Z \mid B^{\perp}} 2 V\left(Z^{x}, B\right)+V_{2}(B) d x \\
& \leq(\operatorname{diam} Z)^{n-2} \cdot\left(2 V\left((\operatorname{diam} Z) B^{2}, B\right)+V_{2}(B)\right) \\
& =(\operatorname{diam} Z)^{n-1} \cdot 2 V_{1}(B)+(\operatorname{diam} Z)^{n-2} V_{2}(B)
\end{aligned}
$$

a.s., we conclude

$$
\mathbb{E} V_{n}\left(Z_{B}+B\right)<\infty \text { and } \mathbb{E}\left[\left(V_{n}\left(Z_{B}+B\right)-V_{n}\left(Z_{B}\right)\right) \cdot(\operatorname{diam} Z) / \rho_{B}\right]<\infty .
$$


Theorem 5.3. Let $N$ be an $\mathbb{N}^{+}$-valued random variable with $\mathbb{E} N<\infty,\left(X_{i}\right)_{i \in \mathbb{N}^{+}}$ an i.i.d. sequence of $\mathbb{R}^{2}$-valued random variables and $\left(Z_{i}\right)_{i \in \mathbb{N}^{+}}$an i.i.d. sequence of random bodies in $\mathbb{R}^{2}$ with $0 \in Z_{i}$ a.s. for all $i \in \mathbb{N}^{+}$. Assume that $\left(X_{i}\right)_{i \in \mathbb{N}^{+}},\left(Z_{i}\right)_{i \in \mathbb{N}^{+}}$ and $N$ are independent and at least one of the following two conditions is satisfied:

(a) The distribution of $X_{1}$ is not concentrated on a set of Lebesgue measure 0.

(b) The distribution of $X_{1}$ is not concentrated on a single point and the probability that $Z_{1}$ is a strictly convex body is positive.

Put $Z:=\bigcup_{i=1}^{N}\left(X_{i}+Z_{i}\right)$. If $Z$ is convex with probability 1 , then $N=1$ a.s.

As a first step preparing the proof of Theorem 5.3 we define for every $x=$ $\left(x^{1}, x^{2}\right) \in \mathbb{R}^{2}$ and $\epsilon>0$ the set

$$
W_{\epsilon}(x):=\left\{\left(y^{1}, y^{2}\right) \in \mathbb{R}^{2} \mid y^{1} \geq x^{1}-\epsilon, y^{2} \in\left[x^{2}-\epsilon, x^{2}\right]\right\} .
$$

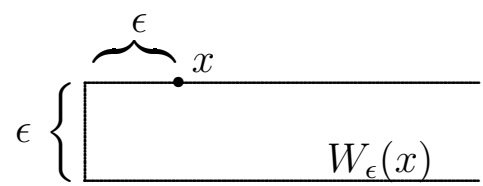

Lemma 5.4. Let $\epsilon>0$ and $A \subseteq \mathbb{R}^{2}$. Then there is a countable subset $\tilde{A} \subseteq A$ with

$$
A \subseteq \bigcup_{p \in \tilde{A}} W_{\epsilon}(p)
$$

Proof. Let $R \in \mathbb{N}^{+}$. We construct a countable set $A_{R} \subseteq A \cap[-R, R]^{2}$ with

$$
A \cap[-R, R]^{2} \subseteq \bigcup_{p \in A_{R}} W_{\epsilon}(p)
$$

In order to do this, we recursively define a sequence $\left(A_{R}^{i}\right)_{i \in \mathbb{N}}$ of subsets of $A \cap$ $[-R, R]^{2}$. Put $A_{R}^{0}:=\emptyset$. For $i \in \mathbb{N}^{+}$consider the set

$$
E_{R}^{i}:=\left\{y^{2} \mid\left(y^{1}, y^{2}\right) \in A \cap[-R, R]^{2} \backslash \bigcup_{p \in A_{R}^{i-1}} W_{\epsilon}(p)\right\}
$$

If it is empty, put $A_{R}^{i}:=A_{R}^{i-1}$. If it has a maximum, choose a point $\left(q^{1}, q^{2}\right) \in$ $A \cap[-R, R]^{2} \backslash \bigcup_{p \in A_{R}^{i-1}} W_{\epsilon}(p)$ such that $q^{2}$ equals this maximum and put $A_{R}^{i}:=A_{R}^{i-1} \cup$ $\left\{\left(q^{1}, q^{2}\right)\right\}$. If it is not empty and has no maximum, choose a sequence $\left(\left(q_{j}^{1}, q_{j}^{2}\right)\right)_{j \in \mathbb{N}}$ in $A \cap[-R, R]^{2} \backslash \bigcup_{p \in A_{R}^{i-1}} W_{\epsilon}(p)$ with $\left(q_{j}^{2}\right)_{j \in \mathbb{N}}$ converging to sup $E_{R}^{i}$, which exists, since $E_{R}^{i} \subseteq[-R, R]$. Then put $A_{R}^{i}:=A_{R}^{i-1} \cup\left\{\left(q_{j}^{1}, q_{j}^{2}\right) \mid j \in \mathbb{N}\right\}$.

For a real number $t$ set $\lceil t\rceil:=\min \{z \in \mathbb{Z} \mid z \geq t\}$ and put $N:=\left\lceil\frac{2 R}{\epsilon}\right\rceil+1$. For $i \in \mathbb{N}^{+}$put $e_{i}:=\left\lceil\left(\sup E_{i}\right) / \epsilon\right\rceil$, where $\sup \emptyset:=-R$. Let $k \in \mathbb{N}^{+}$. In order to show

$$
e_{k+N}<e_{k} \quad \text { or } \quad E_{R}^{k+N}=\emptyset
$$


consider for each $i \in \mathbb{N}^{+}$the set

$$
F_{R}^{i}:=\left\{y^{1} \mid\left(y^{1}, y^{2}\right) \in A \cap[-R, R]^{2} \backslash \bigcup_{p \in A_{R}^{i-1}} W_{\epsilon}(p), y^{2} \geq \epsilon\left(e_{i}-1\right)\right\} .
$$

Obviously $E_{R}^{i}, i \in \mathbb{N}^{+}$, is empty, iff $F_{R}^{i}$ is empty. For $i \in \mathbb{N}^{+}$it is clear from the definition of $A_{R}^{i}$, that neither in the case that $E_{R}^{i}$ has a maximum, nor in the case $E_{R}^{i}$ is not empty and has no maximum, the relations $e_{i+1}=e_{i}$ and $\sup F_{R}^{i+1}>\sup F_{R}^{i}-\epsilon$ can hold at the same time. Hence for all $i \in \mathbb{N}^{+}$we have either $e_{i+1}<e_{i}$, sup $F_{R}^{i+1} \leq$ $\sup F_{R}^{i}-\epsilon$ or $E_{R}^{i+1}=\emptyset$. Since the sequence $\left(e_{i}\right)_{i \in \mathbb{N}^{+}}$is monotonically decreasing, we get by induction, that for each $j \in \mathbb{N}^{+}$one of the relations $e_{k+j}<e_{k}, \sup F_{R}^{k+j} \leq$ $\sup F_{R}^{k}-j \epsilon$ or $E_{R}^{k+j}=\emptyset$ holds. Since $-R \leq \sup F_{R}^{i} \leq R$ for all $i \in \mathbb{N}$, this implies (19). Hence $E_{R}^{i}=\emptyset$ for all $i>N^{2}$ and thus $A_{R}:=A_{R}^{N^{2}}$ fulfills (18).

Now $\tilde{A}:=\bigcup_{R \in \mathbb{N}^{+}} A_{R}$ is a countable subset of $A$ with (17).

Lemma 5.5. Let $X=\left(X^{1}, X^{2}\right)$ be a random vector in $\mathbb{R}^{2}$. Then with probability 1 for each $\epsilon>0$

$$
\mathbb{P}\left(X^{1}-\epsilon \leq Y^{1}, Y^{2} \in\left[X^{2}-\epsilon, X^{2}\right] \mid X\right)>0
$$

holds, where $Y=\left(Y^{1}, Y^{2}\right)$ is a random vector independent of $X$ with the same distribution.

Proof. Let $P$ denote the image measure of $\mathbb{P}$ under $X$ resp. $Y$. We have to show

$$
P\left(\left\{x \in \mathbb{R}^{2} \mid \forall_{\epsilon>0}: P\left(W_{\epsilon}(x)\right)>0\right\}\right)=1,
$$

which is equivalent to

$$
P\left(\left\{x \in \mathbb{R}^{2} \mid \exists_{\epsilon>0}: P\left(W_{\epsilon}(x)\right)=0\right\}\right)=0 .
$$

Since

$$
\left\{x \in \mathbb{R}^{2} \mid \exists_{\epsilon>0}: P\left(W_{\epsilon}(x)\right)=0\right\}=\bigcup_{n=1}^{\infty}\left\{x \in \mathbb{R}^{2} \mid P\left(W_{1 / n}(x)\right)=0\right\}
$$

and $P$ is $\sigma$-additive, it suffices to prove that

$$
A_{n}:=\left\{x \in \mathbb{R}^{2} \mid P\left(W_{1 / n}(x)\right)=0\right\}
$$

is a $P$-zero set for all $n \in \mathbb{N}^{+}$. By the $\sigma$-additivity of $P$ this follows from Lemma 5.4.

Proof of Theorem 5.3. We assume $\mathbb{P}(N \geq 2)>0$ and condition on $N \geq 2$ and $N$.

Let $A$ denote the event that there are $i, k \in\{1, \ldots, N\}$ with $X_{i} \neq X_{k}$, and for all $i, k \in\{1, \ldots, N\}$ with $X_{i} \neq X_{k}$ neither conv $Z_{i}$ nor conv $Z_{k}$ has an edge parallel to $X_{i}-X_{k}$. Now we will show that $A$ has positive probability. If condition (a) is fulfilled, we let $G_{i, k}$ for $i, k \in\{1, \ldots, N\}$ denote the union of all lines passing through $X_{i}$, which are parallel to an edge of conv $Z_{i}$ or conv $Z_{k}$. Now $A$ occurs, if for each $k \in\{2, \ldots, N\}$ the point $X_{k}$ is not in $\bigcup_{i=1}^{k-1} G_{i, k}$, which consists of a most countably many lines (see e.g. [8, Theorem 2.2.5]), and hence is a Lebesgue-zero set. Since $X_{k}$ is independent of $X_{1}, \ldots, X_{k-1}$ for each $k \in\{2, \ldots, N\}$ and its distribution is not 
concentrated on a Lebesgue-zero set, the probability of $A$ is positive. If condition (b) is fulfilled, then with positive probability $Z_{1}, \ldots, Z_{N}$ are all strictly convex and $X_{1} \neq X_{2}$. Hence $\mathbb{P}(A)>0$.

The statements in the following are always meant a.s. conditioned on $N \geq 2$, $N$ and $A$. We can switch the numbers $1, \ldots, N$ in such a way that $X_{1}$ and $X_{2}$ are endpoints of an edge of $\operatorname{conv}\left\{X_{1}, \ldots, X_{N}\right\}$ and hence there is a random unit vector $u \in S^{1}$ with

$$
\left\langle X_{1}, u\right\rangle=\left\langle X_{2}, u\right\rangle \geq\left\langle X_{k}, u\right\rangle, \quad k \in\{3, \ldots, N\} .
$$

It will be proven in Lemma A.9 that this renumbering can be done in a measurable way. Obey that $X_{1}, \ldots, X_{N}$ are not independent and indentically distributed any more, but $Z_{1}, \ldots, Z_{N}$ still are. From now on we additionally condition on $X_{1}$ and $X_{2}$. We put $v:=\frac{X_{2}-X_{1}}{\left\|X_{2}-X_{1}\right\|}$. Let $H_{1}$ and $H_{2}$ denote the points from $X_{1}+Z_{1}$ resp. $X_{2}+Z_{2}$, that have the biggest scalar product with $u$. By $A$ they are determined uniquely. Moreover, $A$ implies that there is a number $\epsilon \in\left(0,\left\|X_{2}-X_{1}\right\|\right)$, for which the event $B$, that $X_{1}+Z_{1}$ does not intersect the segment $\left[H_{1}+\left(\left\|X_{2}-X_{1}\right\|-\epsilon\right) v\right.$, $\left.H_{1}+\left(\left\|X_{2}-X_{1}\right\|-\epsilon\right) v-\epsilon u\right]$, has positive probability.

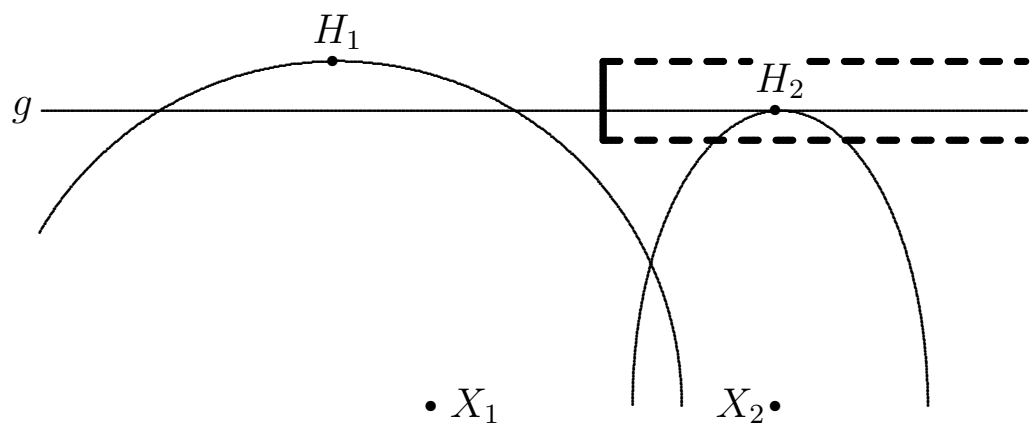

The event $B$ is, that the left body $X_{1}+Z_{1}$ does not intersect the segment ploted thickly. The event $C$ is, that $H_{2}$ lies in the area bounded by the thick lines.

Due to Lemma 5.5 the probability of the event $C$, that

$$
\left\langle H_{1}, u\right\rangle-\epsilon \leq\left\langle H_{2}, u\right\rangle \leq\left\langle H_{1}, u\right\rangle \quad \text { and } \quad\left\langle H_{1}-X_{1}, v\right\rangle-\epsilon \leq\left\langle H_{2}-X_{2}, v\right\rangle
$$

hold, is positive, if we condition (additionally) on $H_{1}$. The events $B$ and $C$ take place at the same time with positive probability. But then the intersection of the line $g$ through $H_{2}$ orthogonal to $u$ and $\left(Z_{1}+X_{1}\right) \cup\left(Z_{2}+X_{2}\right)$ is not connected.

Let $i \in\{3, \ldots, N\}$. Since $Z_{i}$ has the same distribution as $Z_{2}$, the event $D_{i}$, that

$$
\max \left\{\langle x, u\rangle \mid x \in Z_{i}\right\} \leq \max \left\{\langle x, u\rangle \mid x \in Z_{2}\right\},
$$

occurs with positive probability. Moreover, $\left\langle X_{i}, u\right\rangle \leq\left\langle X_{2}, u\right\rangle$. If equality holds, the vectors $X_{i}-X_{1}$ and $X_{i}-X_{2}$, which are not both 0 , are scalar multiples of $v$ and so conv $Z_{i}$ has no edge parallel to $v$, since $A$ is assumed to occure. This means however, that the set

$$
\left\{x \in Z_{i} \mid\langle x, u\rangle=\left\langle H_{2}-X_{2}, u\right\rangle\right\}
$$


consists of at most one point. So, if $D_{i}$ occurs, the intersection of $g$ with $X_{i}+Z_{i}$ consists of at most one element. By the independence of $Z_{1}, \ldots, Z_{n}$ the events $B, C, D_{3}, \ldots, D_{N}$ occure with positive probability at the same time. But then the intersection of $g$ and $\bigcup_{i=1}^{N}\left(X_{i}+Z_{i}\right)$ is not connected and thus $Z$ is not convex.

But this means that the probability (without conditioning) that $Z$ is not convex, is positive, too. Hence the assumption $\mathbb{P}(N \geq 2)>0$ is wrong.

Corollary 5.6. Assume that $\mathbb{E}\left\|X_{1}\right\|^{2}<\infty$ and $\mathbb{E}\left(\operatorname{diam} Z_{1}\right)^{2}<\infty$ hold in addition to the conditions of Theorem 5.3. If $\mathbb{E} V_{2}(Z+r B)$ is a polynomial for some disc body $B \subseteq \mathbb{R}^{2}$, then $N=1$ a.s.

Proof. If $\mathbb{E} V_{2}(Z+r B)$ is a polynomial for some disc body $B \subseteq \mathbb{R}^{2}$, then $Z$ is a.s. convex by Theorem 4.1, whose integrability conditions are satisfied by Theorem 5.1. But then Theorem 5.3 implies $N=1$ a.s.

\section{Outlook}

It is natural to ask for the speed of convergence in Theorem 3.1. If the gauge body has a ball as summand, the answer follows from the following more general theorem (for the notion of a summand see e.g. [8, sections 3.1 and 3.2]).

Theorem 6.1. Let $1<d \leq n$. Let $R$ be an $\mathbb{R}_{0}^{+}$-valued random variable and $S$ and $G$ two $[1, \infty)$-valued random variables. Let $X \subseteq \mathbb{R}^{n}$ be a random body and $Y \subseteq \mathbb{R}^{n}$ a d-dimensional random convex body such that $\operatorname{diam} X<G$, $\operatorname{diam} Y<S$ a.s. and $Y$ contains a.s. a d-dimensional ball of radius $R$ as summand. If

$$
c:=d 2^{d+2} \kappa_{d} \kappa_{n-d} \mathbb{E}\left[\frac{S^{d} \cdot G^{n+1}}{R^{3}}\right]<\infty
$$

then we have

$$
\mathbb{E}\left[V_{n}\left(X_{Y}+r Y\right)-V_{n}(X+r Y)\right]<c \cdot r^{d-3}, \quad r>1 .
$$

This is Satz 2.15 of the Ph.D. thesis [5]. There will be an article soon.

\section{A Measurability}

We let af ${ }^{-}: \mathcal{C} \rightarrow \mathcal{F}$ denote the map which asigns to a body the linear space parallel to its affine hull.

Lemma A.1. The map af $\mathrm{f}^{-}: \mathcal{C} \rightarrow \mathcal{F}$ is lower semicontinuous, when $\mathcal{C}$ is equipped with the Hausdorff topology and $\mathcal{F}$ with the topology of closed convergence.

Proof. Let $\left(K_{k}\right)_{k \in \mathbb{N}^{+}}$be a sequence of bodies converging to a body $K$. In order to show af $K \subseteq \lim _{k \rightarrow \infty}$ af $^{-} K_{k}$, let $y \in$ af $^{-} K$. Then there are numbers $\lambda_{1}, \ldots, \lambda_{n} \in \mathbb{R}$ and points $y_{1}, \ldots, y_{n} \in K$ such that $y=\lambda_{1} y_{1}+\cdots+\lambda_{n} y_{n}$ and $\lambda_{1}+\cdots+\lambda_{n}=0$. Now there are sequences $\left(y_{1, k}\right)_{k \in \mathbb{N}^{+}}, \ldots,\left(y_{n, k}\right)_{k \in \mathbb{N}^{+}}$converging to $y_{1}, \ldots, y_{n}$ such that $y_{1, k}, \ldots, y_{n, k} \in K_{k}$ for all $k \in \mathbb{N}^{+}$. So $y_{\cdot, k}:=\lambda_{1} y_{1, k}+\cdots+\lambda_{n} y_{n, k} \in$ af $^{-} K_{k}$ for $k \in \mathbb{N}^{+}$ and $\lim _{k \rightarrow \infty} y_{\cdot, k}=y$. 
We let $\mathcal{L}_{j}^{n}$ denote the set of all $j$-dimensional linear subspaces of $\mathbb{R}^{n}$.

Corollary A.2. The set $\mathcal{K}_{l}$ of all bodies of $\mathbb{R}^{n}$, whose affine hull has at most dimension $l$, is closed (w.r.t. the Hausdorff metric) for $l \in\{0, \ldots, n\}$.

Proof. The set $\mathcal{L}:=\cup_{j=0}^{l} \mathcal{L}_{j}^{n}$ is a finite union of compact sets and hence compact. Let $\left(K_{k}\right)_{k \in \mathbb{N}}^{+}$be a sequence of bodies, whose affine hull has at most $l$ dimensions, converging to a body $K$. Then af ${ }^{-} K \subseteq \lim _{k \rightarrow \infty}$ af $^{-} K_{k}$ by Lemma A.1. So af $K$ has at most dimension $l$.

Lemma A.3. The set $\mathcal{K}_{2}^{\|}$of all pairs of convex bodies $(K, B)$ lying in two 2dimensional parallel affine subspaces of $\mathbb{R}^{n}$ is a closed subset of $\mathcal{C} \times \mathcal{C}$.

Proof. Let $\left(K_{k}\right)_{k \in \mathbb{N}^{+}}$and $\left(B_{k}\right)_{k \in \mathbb{N}^{+}}$be sequences of convex bodies converging to bodies $K$ and $B$ such that for every $k \in \mathbb{N}^{+}$the bodies $K_{k}$ and $B_{k}$ lie in parallel 2-dimensional affine subspaces. For each $k \in \mathbb{N}^{+}$choose $x_{k} \in K_{k}, y_{k} \in B_{k}$ and orthogonal unit vectors $u_{k}$ and $v_{k}$ such that

$$
K_{k} \subseteq\left\{x_{k}+\lambda u_{k}+\mu v_{k} \mid \lambda, \mu \in \mathbb{R}\right\} \quad \text { and } \quad B_{k} \subseteq\left\{y_{k}+\lambda u_{k}+\mu v_{k} \mid \lambda, \mu \in \mathbb{R}\right\} .
$$

Due to compactness arguments we may assume that the sequences $\left(x_{k}\right)_{k \in \mathbb{N}^{+}},\left(y_{k}\right)_{k \in \mathbb{N}^{+}}$, $\left(u_{k}\right)_{k \in \mathbb{N}^{+}}$and $\left(v_{k}\right)_{k \in \mathbb{N}^{+}}$converge to limits $x, y, u$ and $v$. Now for each point $p \in K$ there is a sequence $\left(p_{k}\right)_{k \in \mathbb{N}^{+}}$with $p_{k} \in K_{k}$ for each $k \in \mathbb{N}^{+}$converging to $p$ and hence

$$
p=\lim _{k \rightarrow \infty} x_{k}+\left\langle p_{k}-x_{k}, u_{k}\right\rangle u_{k}+\left\langle p_{k}-x_{k}, v_{k}\right\rangle v_{k}=x+\langle p-x, u\rangle u+\langle p-x, v\rangle v .
$$

Thus $K \subseteq\{x+\lambda u+\mu v \mid \lambda, \mu \in \mathbb{R}\}$ and similar $B \subseteq\{y+\lambda u+\mu v \mid \lambda, \mu \in \mathbb{R}\}$.

Corollary A.4. The set $\mathcal{K}^{2 \|}$ is measurable.

Proof. Since

$$
\mathcal{K}^{2 \|}=\mathcal{K}_{2}^{\|} \backslash\left(\mathcal{K}_{1} \times \mathcal{K}\right) \backslash\left(\mathcal{K} \times \mathcal{K}_{1}\right)
$$

the assertion is implied by Corollary A.2 and Lemma A.3.

For $j \in \mathbb{N}^{+}$we let $\mathcal{A}_{j}$ denote the set of all 2-dimensional convex bodies $K$ which have a boundary point with two exterior normal unit vectors lying in af ${ }^{-} K$ and forming an angel of at least $1 / j$.

Lemma A.5. The sets $\mathcal{A}_{j}, j \in \mathbb{N}^{+}$, are closed in $\mathcal{C}$.

Proof. Let $\left(K_{k}\right)_{k \in \mathbb{N}^{+}}$be a sequence in $\mathcal{A}_{j}$ converging to $K \in \mathcal{C}$. Since $\mathcal{K}$ is closed (as shown in the proof of [9, Theorem 2.4.2]), we have $K \in \mathcal{K}$. By Corollary A.2 $K$ is 2-dimensional. Now there is a point $x_{k} \in \mathrm{bd} K_{k}$ with exterior unit normal vectors $u_{k}$ and $v_{k}$ lying in $\mathrm{af}^{-} K_{k}$ and having an angel of at least $1 / j$ for every $k \in \mathbb{N}^{+}$. We may assume that the sequences $\left(x_{k}\right)_{k \in \mathbb{N}^{+}},\left(u_{k}\right)_{k \in \mathbb{N}^{+}}$and $\left(v_{k}\right)_{k \in \mathbb{N}^{+}}$converge to limits $x, u$ and $v$. Obviously $x$ has exterior unit normal vectors $u$ and $v$ and these vectors lie both in af $^{-} K$ and form an angel of at least $1 / j$. Hence $K \in \mathcal{A}_{1 / j}$.

Corollary A.6. The set of all disc bodies $\mathcal{K}_{\text {Disc }}$ is measurable. 
Proof. The set of all disc bodies is the intersection of the set of all 2-dimensional bodies with $\mathcal{K}$ minus $\cup_{j=1}^{\infty} \mathcal{A}_{j}$. Hence it is measurable.

Lemma A.7. The $\operatorname{map} \mathcal{C} \times \mathcal{C} \rightarrow \mathcal{C},(K, B) \mapsto K_{B}$ is measurable.

Proof. By [3, Lemma 2.2] the map

$$
F: \mathcal{C} \times \mathcal{L}_{l}^{n} \rightarrow \mathcal{C},(K, E) \mapsto \bigcup_{x \in E^{\perp}} \operatorname{conv}((x+E) \cap K)
$$

is measurable for all $l \in\{0, \ldots, n\}$. Since $K_{B}=F\left(K\right.$, af $\left.f^{-} B\right)$ for all $K, B \in \mathcal{C}$, the statement follows from Lemma A.1.

Recall that $\mathcal{K}_{00}$ denotes the set of all convex bodies $K$ with $0 \in \operatorname{int} K$.

Lemma A.8. The map $w: \mathcal{K}_{00} \times \mathbb{R}_{0}^{+} \rightarrow \mathbb{R}_{0}^{+}$is lower semicontinuous.

Proof. Let $\left(B_{k}\right)_{k \in \mathbb{N}^{+}}$be a sequence in $\mathcal{K}_{00}$ converging to $B \in \mathcal{K}_{00}$ and $\left(r_{k}\right)_{k \in \mathbb{N}^{+}}$a sequence in $\mathbb{R}_{0}^{+}$converging to $r \in \mathbb{R}_{0}^{+}$. Assume that $\left(w_{B_{k}}\left(r_{k}\right)\right)_{k \in \mathbb{N}^{+}}$has an accumulation point less than $w_{B}(r)$. Then there is a subsequence, w.l.o.g. the sequence itself, converging to this point. For every $k \in \mathbb{N}^{+}$there are points $y_{k} \in B^{d}$ and $z_{k} \in \mathbb{R}^{d}$ such that $d_{B_{k}}\left(0, z_{k}\right)=r_{k}, y_{k} \in \Pi_{B_{k}}\left(0 y_{k}, z_{k}\right)$ and $d_{B_{k}}\left(y_{k}, z_{k}\right)=w_{B_{k}}\left(r_{k}\right)$. Since $\left(B_{k}\right)_{k \in \mathbb{N}^{+}}$and $\left(r_{k}\right)_{k \in \mathbb{N}^{+}}$converge, there are $S, s \in \mathbb{R}_{0}^{+}$such that $B_{k} \subseteq S B^{d}$ and $r_{k} \leq s$ for all $k \in \mathbb{N}^{+}$. Hence $z_{k} \in s S B^{d}$ for all $k \in \mathbb{N}^{+}$and so we may assume that the sequences $\left(z_{k}\right)_{k \in \mathbb{N}^{+}}$and $\left(y_{k}\right)_{k \in \mathbb{N}^{+}}$converge to points $z$ and $y$. Now the continuity of $d_{B}$ implies $d_{B}(0, z)=r$ and $y \in \Pi_{B}(0 y, z)$. Thus

$$
w_{B}(r) \leq d_{B}(y, z)=\lim _{k \rightarrow \infty} d_{B_{k}}\left(y_{k}, z_{k}\right)=\lim _{k \rightarrow \infty} w_{B_{k}}\left(r_{k}\right),
$$

contradicting the assumption. So $w$ is lower semicontinuous.

Lemma A.9. There is a measurable map $f=\left(f_{1}, \ldots, f_{n}\right):\left(\mathbb{R}^{2}\right)^{n} \rightarrow\left(\mathbb{R}^{2}\right)^{n}$ such that for each $\hat{x}=\left(x_{1}, \ldots, x_{n}\right) \in\left(\mathbb{R}^{2}\right)^{n}$ there is a bijection $\sigma:\{1, \ldots, n\} \rightarrow\{1, \ldots, n\}$ with $f_{j}(\hat{x})=x_{\sigma(j)}$ for $j=1, \ldots, n$ and a vector $u \in \mathbb{R}^{2}$ with

$$
\left\langle f_{1}(\hat{x}), u\right\rangle=\left\langle f_{2}(\hat{x}), u\right\rangle \geq\left\langle f_{j}(\hat{x}), u\right\rangle, \quad j=3, \ldots, n .
$$

Proof. We choose $f_{1}(\hat{x})$ to be the lower tangent point (see $\left[9\right.$, p. 110]) of $\left\{x_{1}, \ldots, x_{n}\right\}$. If $P(\hat{x}):=\left\{x_{1}, \ldots, x_{n}\right\} \backslash\left\{f_{1}(\hat{x})\right\}$ is the empty set, put $f_{j}(\hat{x}):=x_{1}$ for all $j \in$ $\{2, \ldots, n\}$. Else choose $f_{2}(\hat{x})$ to be the point $p \in P$, for which $\left\langle p-f_{1}(\hat{x}), e_{1}\right\rangle / \| p-$ $f_{1}(\hat{x}) \|$ is maximal; in case there is more than one maximum, choose the one for which $\left\|p-f_{1}(\hat{x})\right\|$ is larger. For $j \in\{3, \ldots, n\}$ put $f_{j}(\hat{x}):=x_{j-k_{j}}$, where

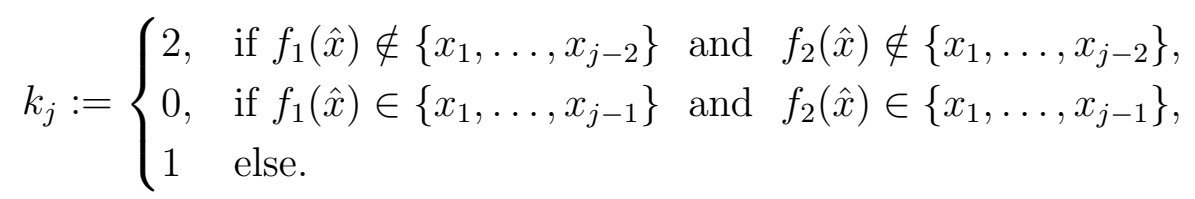

Now the measurability of $f_{1}$ can be established using semicontinuity arguments. In order to proof in the case $n>1$ that $f_{2}$ is measurable, we notice, that the sets

$$
\begin{aligned}
R & :=\left\{\hat{x} \in\left(\mathbb{R}^{2}\right)^{n} \mid P(\hat{x})=\emptyset\right\}, \\
R_{1} & :=\left\{\hat{x} \in\left(\mathbb{R}^{2}\right)^{n} \backslash R \mid \exists_{i \in\{1, \ldots, n\}}:\left(f_{1}(\hat{x}) \neq x_{i} \wedge\left\langle f_{1}(\hat{x}), e_{2}\right\rangle=\left\langle x_{i}, e_{2}\right\rangle\right)\right\} \text { and } \\
R_{2} & :=\left\{\hat{x} \in\left(\mathbb{R}^{2}\right)^{n} \backslash R \mid \forall_{i \in\{1, \ldots, n\}}:\left(f_{1}(\hat{x})=x_{i} \vee\left\langle f_{1}(\hat{x}), e_{2}\right\rangle \neq\left\langle x_{i}, e_{2}\right\rangle\right)\right\}
\end{aligned}
$$


are measurable. Further we consider the functions

$$
\begin{array}{ll}
g_{1}: R_{1} \cup R_{2} \rightarrow \mathbb{R}, & \hat{x} \mapsto \frac{\left\langle f_{2}(\hat{x})-f_{1}(\hat{x}), e_{1}\right\rangle}{\left\|f_{2}(\hat{x})-f_{1}(\hat{x})\right\|} \\
g_{2}: R_{1} \cup R_{2} \rightarrow \mathbb{R}, & \hat{x} \mapsto\left\|f_{2}(\hat{x})-f_{1}(\hat{x})\right\| .
\end{array}
$$

Their restrictions to $R_{1}$ and $R_{2}$ are semincontinuous respectively and hence both functions are measurable. Since $f_{1}(\hat{x})=f_{2}(\hat{x})$ for all $\hat{x} \in R$, we have shown the measurability of $f_{2}$.

The measurability of $f_{j}, j \in\{3, \ldots, n\}$, is easy to see. Obviously $f$ has also the other properties stated in the lemma.

\section{References}

[1] Cohn, D.L.: Measure Theory, Birkhäuser, Boston, 1980

[2] Heveling, M., Hug, D., Last, G.: Does polynomial parallel volume imply convexity?, Mathematische Annalen 328, 2004, 469-479

[3] Hug, D., Last, G., Weil, W.: Polynomial parallel volume, convexity and contact distributions of random sets, Probability Theory and Related Fields 135, 2006, $169-200$

[4] Kampf, J.: On weighted parallel volumes, Beiträge zur Algebra und Geometrie, 50, 2009, 495-519

[5] Kampf, J.: Das Parallelvolumen und abgeleitete Funktionale, Ph.D. thesis 2009, University of Karlsruhe

[6] Kneser, M.: Über den Rand von Parallelkörpern, Mathematische Nachrichten 5, 1951, 241-251

[7] Lusternik, L.: Die Brunn-Minkowskische Ungleichung für beliebige messbare Mengen, Comptes Rendus (Doklady) de l'Academie des Sciences de l'URSS 3, $1935,55-58$

[8] Schneider, R.: Convex Bodies: The Brunn-Minkowski Theory, Cambrigde University Press, 1993

[9] Schneider, R., Weil, W.: Stochastic and Integral Geometry, Springer, Berlin, Heidelberg, 2008

[10] Steiner, J.: Über parallele Flächen, 1840, abgedruckt in: Weierstrass (Hrsg.): Jacob Steiner's gesammelte Werke, Chelsea Publishing Company, Bronx, New York, 1882, Band II, 171-176

[11] Sz.-Nagy, B.: Über Parallelmengen nichtkonvexer ebener Bereiche, Acta Scientiarum Mathematicarum 20, 1959, 36-47 